\title{
Singularity-free defect mechanics for polar media
}

Received: 8 January 2019 / Accepted: 12 May 2019 / Published online: 21 May 2019

(C) The Author(s) 2019

\begin{abstract}
We present singularity-free solution for cracks within polar media in which material points possess both position and orientation. The plane strain problem is addressed in this study for which the generalized continua including micropolar, nonlocal micropolar, and gradient micropolar elasticity theories are employed. For the first time, the variationally consistent boundary conditions are derived for gradient micropolar elasticity. Moreover, having reviewed the solution to line defects including glide edge dislocation and wedge disclination from the literature, the fields of a climb edge dislocation within micropolar, nonlocal micropolar and gradient micropolar elasticity are derived. This completes the collection of line defects needed for an inplane strain analysis. Afterward, as the main contribution, using both types of line defects (i.e., dislocation being displacement discontinuity and disclination being rotational discontinuity), the well-established dislocation-based fracture mechanics is systematically generalized to the dislocation- and disclination-based fracture mechanics of polar media for which we have three translations together with three rotations as degrees of freedom. Due to the application of the line defects, incompatible elasticity is employed throughout the paper. Cracks under three possible loadings including stress and couple stress components are analyzed, and the corresponding line defect densities and stress and couple stress fields are reported. The singular fields are obtained once using the micropolar elasticity, while nonlocal micropolar, and gradient micropolar elasticity theories give rise to singularity-free fracture mechanics.
\end{abstract}

Keywords Crack - Inplane - Dislocation - Disclination · Micropolar elasticity · Gradient micropolar elasticity $\cdot$ Nonlocal micropolar elasticity $\cdot$ Nonsingular

\section{Introduction}

Generalized (or extended) continua include (strong) nonlocal theories as well as higher-order (or microcontinuum) and higher-grade (or weak nonlocal) extensions to the classical continuum theories. Within nonlocal elasticity, nonlocal stresses at a material point is expressed as a function of weighted values of the entire stress field. On the other hand, by increasing the order of the theory, microcontinuum field theories (e.g., micromorphic elasticity, microstretch elasticity, micropolar elasticity, and dilatation elasticity) are obtained. Further, higher-grade versions include gradient elasticity theories.

A microcontinuum is a continuous collection of deformable point particles [14] for which the stress measures include force stress as well as couple stress [13,54,55]. Microcontinuum field theories address media for which the microstructure is no longer rigid (e.g., polar media). These theories are in fact a two-level continuum models where macroscopic deformation field is enhanced with the microscopic deformation. Within

Communicated by Victor Eremeyev, Holm Altenbach.

S. M. Mousavi $(\varangle)$

Department of Engineering and Physics, Karlstad University, 65188 Karlstad, Sweden

E-mail: mahmoud.mousavi@kau.se 
micromorphic elasticity theory, material points possess twelve degrees of freedom including a translation vector (three classical translational degrees of freedom) and a microdeformation tensor (as nine microstructural deformation being micro-rotation, micro-shear, and micro-dilatation). Restricting the degrees of freedom, the microstretch elastic solid is obtained that addresses seven degrees of freedom (including three translations, three micro-rotations, and one micro-stretch). Constraining further, micropolar elasticity is governed which possesses three translations together with three micro-rotations. Finally, dilatation elasticity is formulated by considering material points with four degrees of freedom (including three translations and one dilatation). In addition to these classical microcontinuum theories, recently other models have been formulated (e.g., relaxed micromorphic continuum [16]).

Within micropolar elasticity $[8,12]$ (also denoted as general or unconstrained Cosserat theory), each point has six degrees of freedom, while the couple stress elasticity (constrained or restricted Cosserat theory) possesses constrained micro-rotations resulting only in three translational degrees of freedom. Due to asymmetric stress and couple stress tensors, the micropolar elasticity is also named as asymmetric (or nonsymmetric) theory of elasticity [47]. The micropolar, couple stress, and classical elasticity frameworks include six, four, and two elastic constants, respectively.

Generalizing the classical elasticity (with three degrees of freedom) to micropolar elasticity is required to capture physical features of some materials such as granular materials and bone. Once generalizing the continuum toward micropolar theory, we can combine the higher-order extension with the higher-grade extension to achieve gradient micropolar elasticity [29,30]. Moreover, a nonlocal version of the micropolar elasticity can be envisaged as nonlocal micropolar elasticity [31].

In line with advancing the generalized continuum theory for perfect solids, a generalized fracture theory needs also to be established. In this study, we intend to investigate the defects within three different frameworks including micropolar, nonlocal micropolar, and gradient micropolar continua. Within micropolar elasticity and for a crack of mode I, the stress field has a singularity of $r^{-1 / 2}$ (similar to linear elastic fracture mechanics), while couple stress possesses a singularity of $r^{-3 / 2}$ [56]. Such singularity at the crack tip results in an unbounded strain energy density. An unbounded strain energy is unrealistic at the crack tip. Accordingly, generalizing micropolar elasticity toward its nonlocal or gradient versions has been suggested to, if possible, regularize the field quantities such as stress and couple stress tensors. Then, while still six degrees of freedom are considered at each point, the nonlocal or gradient treatment leads to the regularized frameworks giving more realistic simulations of defects. This will provide us a singularity-free defect mechanics for polar media. In plasticity, similar extensions, e.g., based on gradient elasticity of Helmholtz type, have been reported providing singularity-free dislocation dynamics (e.g., [48]). The need for regularized frameworks is confirmed for materials such as silicon by observations at the nanoscale [50], while further experiments are required to shed light on this aspect for polar media.

In the literature, there are disagreements about the stress singularity at the crack tip within micropolar elasticity. Both singularity of $r^{-1 / 2}$ (e.g., $[2,6,10,33,34,52,58]$ ) and singularity of $r^{-3 / 2}$ (e.g., [56]) have been reported for couple stress at the crack tip. Warren and Byskov [56] claimed that the priori assumption on the singularity of the couple stress results in such disagreement. The results from the present approach, i.e., dislocation- and disclination-based method, confirms the singularity of $r^{-1 / 2}$ both for the stress and couple stress components at the crack tip within micropolar elasticity.

The line defects include dislocations as displacement discontinuity and disclinations as rotational discontinuity [9,22]. In contrast to disclinations [61], the mechanics of dislocations has been extensively used for modeling of physical phenomenon (e.g., plasticity $[1,23]$ and fracture $[21,57]$ ) in the mechanics of materials. Dislocations and disclinations have been studied within micropolar elasticity (e.g., see [46,49]), nonlocal micropolar elasticity [28-30], and gradient micropolar elasticity [29-31]. Stemming from the Volterra process picture of a defect [25], the line defects include (edge and screw) dislocations and (twist and wedge) disclinations (originally denoted as disinclination) [9]. Zubov [63] has looked into the continuously distributed dislocations and disclinations within nonlinear micropolar media.

A dislocation- and disclination-based approach serves well toward modeling cracks based on a wellestablished literature of defects mechanics. Dislocation-based fracture mechanics (or distributed dislocation technique) is an efficient and well-established method within classical elasticity [4]. The extension of this method toward a number of generalized continuum theories has been addressed recently. The distributed dislocation technique has been used for the analysis of cracks within a compatible version of couple stress elasticity (restricted Cosserat theory) $[18,19]$. However, dislocations and disclinations are the source of incompatibility. Accordingly, in order to employ the dislocation-based fracture, an incompatible framework should be considered [32]. Considering the incompatibility, the dislocation-based fracture theory has been reported for a 
number of generalized continuum theories including isotropic and anisotropic Helmholtz-type nonlocal elasticity $[39,40]$, gradient elasticity $[38,41,42]$, bi-Helmholtz nonlocal elasticity $[43,44]$, bi-Helmholtz (second) gradient elasticity $[43,44]$. The dislocation-based method has also been used for the asymptotic inplane crack analysis in materials with rotating particles within so-called small-scale micropolar elasticity [11].

Several numerical methods have been used to study cracks within micropolar elasticity. Discrete element method, boundary element method [3,51], extended finite element method [24,59,60], and pseudospectral technique [53] are among those. Comparing the dislocation- and disclination-based approach and other methods, it is understood that once the analytical solution to the line defect is available, the dislocationand disclination-based approach seems to be more efficient and computationally less expensive. Moreover, a dislocation- and disclination-based approach provides the possibility of studying the interaction of dislocations and cracks addressing the full-field solutions.

The purpose of this paper is to analyze cracks within an incompatible framework of linear micropolar elasticity together with its extension to nonlocal and gradient versions. The model consists of two types of length scales (being the Cosserat length scales and gradient/nonlocal length scale), without assuming any relationship between the scales. The full-field solutions are derived which can be simplified to derive asymptotics (e.g., far-field or intermediate solutions). It is noted that in this study, we have not used the model for any specific microstructure, i.e., no attempt is made to calculate the characteristic lengths. Instead of symbolic (direct) tensor notation, a Cartesian frame and indicial notation are employed which suits for the analysis of defect geometries considered here while avoiding uncertainties of definitions of operators.

The paper is organized as follows. Section 2 reviews those generalized continua which will be addressed in this study. Then, a variational formulation is provided in the third section for micropolar as well as gradient micropolar elasticity to derive the consistent boundary conditions. The collection of line defects is then given in Sect. 4. The main part of the paper appears in Sect. 5 addressing the analysis of complex defects based on topological line defects. Cracks with all possible loading cases are studied in Sect. 6 where the defect densities as well as stress and couple stress fields are presented. The paper is then concluded in Sect. 7.

\section{Generalized continua}

Generalized continuum mechanics successes in capturing features that cannot be addressed in a classical continuum theory [36]. The formulation of mechanics of generalized continua is an attempt to bring closer the continuum modeling predictions in one hand and the architectured structure or discrete material behavior on the other hand. Considering the phenomenon and material/structure to be studied, appropriate generalization needs to be selected. However, as expected, in comparison with classical elasticity, the generalized continua have more complicated constitutive equations, and the experimental characterization of their non-classical elastic constants is much more difficult.

In this paper, micropolar elasticity and its generalized versions including nonlocal, and gradient micropolar elasticity are discussed. For this purpose and for completeness, in the following subsections, these frameworks are reviewed from the literature. In order to distinguish them, the superscript ' $c$ ', ' $n$ ', and ' $g$ ' are used for (classical) micropolar, nonlocal micropolar and gradient micropolar elasticity, respectively.

\subsection{Micropolar elasticity}

The notion of linear local (classical) micropolar elasticity theory is briefly reported here. Hereafter, the local micropolar elasticity is simply denoted as micropolar elasticity, and its component is denoted with superscript ' $c$ '. The micropolar elasticity contains two stress measures including (force) stress tensor and couple stress tensor. In this theory and in the absence of body loads (body force and body couple), the equilibrium equations of momentum and of moment of momentum read $[12,29,47]$

$$
\begin{aligned}
& \sigma_{i j, j}^{c}=0, \\
& \mu_{i j, j}^{c}-\epsilon_{i j k} \sigma_{j k}^{c}=0,
\end{aligned}
$$

where the index refers to $x, y$, and $z, \epsilon_{i j k}$ is the permutation (Levi-Civita) symbol, ()$_{, j}=\partial_{j}$, and $\sigma_{i j}^{c}$ and $\mu_{i j}^{c}$ denote the (asymmetric) stress and (asymmetric) couple stress tensors, respectively. As an important feature of the micropolar elasticity, the stress field appears as source to the couple stress field. In contrast to classical elasticity, due to asymmetric stress components, the index order is not arbitrary. Here, we follow the strain measures 

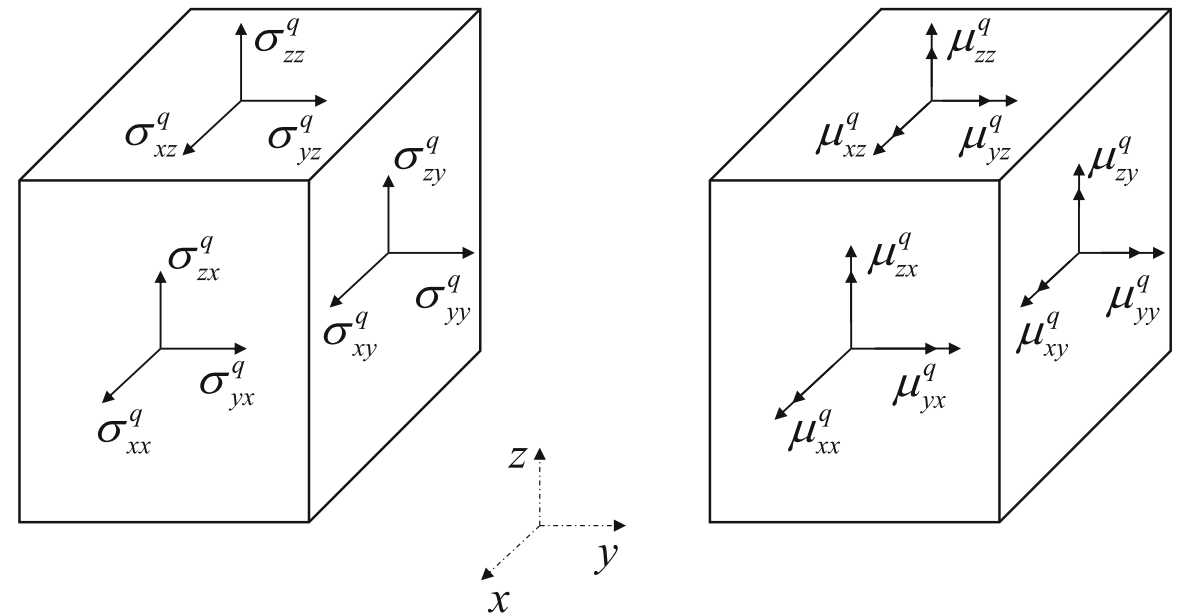

Fig. 1 Stress and couple stress components subscripts, $q \in\{c, n, g\}$

defined in [12,29], while in the literature (e.g., $[15,47])$, the transpose of such definitions are also used. Following Eremeyev et al. [12], in this study, for the stress tensor $\sigma_{i j}^{c}$ (and also couple stress tensor $\mu_{i j}^{c}$ ), the second subscript identifies the area element, whereas the first subscript shows the direction of the stress action (Fig. 1).

These stress tensors are given by the constitutive equations [12,29]

$$
\begin{aligned}
\sigma_{i j}^{c} & =\lambda \delta_{i j} \gamma_{k k}^{c}+(\mu+\eta) \gamma_{i j}^{c}+(\mu-\eta) \gamma_{j i}^{c}, \\
\mu_{i j}^{c} & =\alpha \delta_{i j} \kappa_{k k}^{c}+(\beta+\gamma) \kappa_{i j}^{c}+(\beta-\gamma) \kappa_{j i}^{c} .
\end{aligned}
$$

Here, $\delta_{i j}$ is the Kronecker delta. The deformation measures include the elastic micropolar distortion tensor $\gamma_{i j}^{c}$ and elastic micropolar bend-twist (or wryness) tensor $\kappa_{i j}^{c}$. The classical Lamé material constants $\lambda$ and $\mu$ are accompanied by the Cosserat material constants $\alpha, \beta, \gamma$, and $\eta$ for an isotropic micropolar medium. Note that the nomenclature for these material constants are not unique in the literature. These Cosserat material constants define two characteristic length scale $l_{1}$ and $l_{2}$ as

$$
l_{1}^{2}=\frac{(\mu+\eta)(\beta+\gamma)}{4 \mu \eta}, \quad l_{2}^{2}=\frac{\alpha+2 \beta}{4 \eta} .
$$

It is noted that the condition of positive definite quadratic form for the strain energy density implies restrictions on the material elastic constants.

The inverse form of the constitutive equations (2) reads

$$
\begin{aligned}
& 4 \mu \eta \gamma_{i j}^{c}=-\frac{2 \lambda \eta}{3 \lambda+2 \mu} \delta_{i j} \sigma_{k k}^{c}+(\mu+\eta) \sigma_{i j}^{c}-(\mu-\eta) \sigma_{j i}^{c}, \\
& 4 \beta \gamma \kappa_{i j}^{c}=-\frac{2 \alpha \gamma}{3 \alpha+2 \beta} \delta_{i j} \mu_{k k}^{c}+(\beta+\gamma) \mu_{i j}^{c}-(\beta-\gamma) \mu_{j i}^{c} .
\end{aligned}
$$

Since we wish to address the dislocations and dislocations, an incompatible framework should be used. Within micropolar elasticity, the displacement vector field is accompanied by an independent micro-rotation vector field resulting in a continuum with six degrees of freedom at each point. The total micropolar distortion and total micropolar bend-twist tensors, which can be additively divided into elastic and plastic components, are given by $[14,29]$

$$
\begin{aligned}
& \gamma_{i j}^{c, t}=\gamma_{i j}^{c}+\gamma_{i j}^{c, p}=u_{i, j}^{c}+\epsilon_{i j k} \phi_{k}^{c}, \\
& \kappa_{i j}^{c, t}=\kappa_{i j}^{c}+\kappa_{i j}^{c, p}=\phi_{i, j}^{c} .
\end{aligned}
$$


Here, $u_{i}^{c}$ and $\phi_{i}^{c}$ denote the displacement and micro-rotation vectors, respectively, while $\gamma_{i j}^{c, p}$ and $\kappa_{i j}^{c, p}$ are the plastic micropolar distortion and plastic micropolar bend-twist tensors, respectively. Consequently, within an incompatible micropolar elasticity theory, the elastic deformation measures $\gamma_{i j}^{c}$ and $\kappa_{i j}^{c}$, appeared in the constitutive equations (2), are given by

$$
\begin{aligned}
& \gamma_{i j}^{c}=u_{i, j}^{c}+\epsilon_{i j k} \phi_{k}^{c}-\gamma_{i j}^{c, p}, \\
& \kappa_{i j}^{c}=\phi_{i, j}^{c}-\kappa_{i j}^{c, p} .
\end{aligned}
$$

The (elastic, plastic, total) micropolar strain tensor is the symmetric part of the (elastic, plastic, total) micropolar distortion tensor. Note that while the total deformation measures $\gamma_{i j}^{c, t}$ and $\kappa_{i j}^{c, t}$ are compatible expressions, both the elastic components $\gamma_{i j}^{c}$ and $\kappa_{i j}^{c}$ and their plastic counterparts $\gamma_{i j}^{c, p}$ and $\kappa_{i j}^{c, p}$ are incompatible quantities. The incompatibility, as discussed in Sect. 4, gives rise to the dislocation and disclination densities.

\subsection{Nonlocal micropolar elasticity}

Within the Eringen's nonlocal micropolar elasticity theory [15], the equilibrium equations of momentum and of moment of momentum read

$$
\begin{aligned}
& \sigma_{i j, j}^{n}=0, \\
& \mu_{i j, j}^{n}-\epsilon_{i j k} \sigma_{j k}^{n}=0,
\end{aligned}
$$

where $\sigma_{i j}^{n}$ and $\mu_{i j}^{n}$ denote the nonlocal stress and nonlocal couple stress tensors of micropolar elasticity, respectively. In this context, these components are equivalently referred to as stress and couple stress tensors of nonlocal micropolar elasticity. The nonlocal stress and nonlocal couple stress tensors are given by

$$
\begin{aligned}
& \sigma_{i j}^{n}(\boldsymbol{x})=\int_{V} \alpha(|\boldsymbol{x}-\boldsymbol{y}|) \sigma_{i j}^{c}(\boldsymbol{y}) \mathrm{d} v(\boldsymbol{y}), \\
& \mu_{i j}^{n}(\boldsymbol{x})=\int_{V} \alpha(|\boldsymbol{x}-\boldsymbol{y}|) \mu_{i j}^{c}(\boldsymbol{y}) \mathrm{d} v(\boldsymbol{y}) .
\end{aligned}
$$

Within Eringen's nonlocal micropolar elasticity theory, the kernel function $\alpha(|\boldsymbol{x}-\boldsymbol{y}|)$ is considered to be the Green function of the differential operator $L=1-\epsilon^{2} \Delta$, i.e.,

$$
\left(1-\epsilon^{2} \Delta\right) \alpha(|\boldsymbol{x}-\boldsymbol{y}|)=\delta(\boldsymbol{x}-\boldsymbol{y})
$$

where $\epsilon$ is a characteristic material internal length $(\epsilon \geq 0)$ and $\Delta$ and $\delta$ are the Laplacian and the Dirac delta function, respectively. Accordingly, the integral relations (8) reduce to the inhomogeneous Helmholtz equation

$$
\begin{aligned}
& \left(1-\epsilon^{2} \Delta\right) \sigma_{i j}^{n}=\sigma_{i j}^{c}, \\
& \left(1-\epsilon^{2} \Delta\right) \mu_{i j}^{n}=\mu_{i j}^{c},
\end{aligned}
$$

where the classical stress $\sigma_{i j}^{c}$ and couple stress $\mu_{i j}^{c}$ are the source for the nonlocal stress $\sigma_{i j}^{n}$ and nonlocal couple stress $\mu_{i j}^{n}$, respectively.

It is noted that the deformation measures of nonlocal micropolar elasticity are identical to those of (local) micropolar elasticity. In other words, the nonlocal framework does not include any definition of nonlocal micropolar distortion or nonlocal micropolar bend-twist tensor. 


\subsection{Gradient micropolar elasticity}

The gradient micropolar elasticity theory proposed by Lazar and Maugin [29,30] extends the classical equilibrium equations of micropolar elasticity to

$$
\begin{aligned}
& \left(\sigma_{i j}^{g}-\partial_{k} \tau_{i j k}\right)_{, j}=0, \\
& \left(\mu_{i j}^{g}-\partial_{k} \lambda_{i j k}\right)_{, j}-\epsilon_{i j k}\left(\sigma_{j k}^{g}-\tau_{j k l, l}\right)=0,
\end{aligned}
$$

where $\sigma_{i j}^{g}$ and $\mu_{i j}^{g}$ denote the stress and couple stress tensors of gradient micropolar elasticity, respectively. Further, $\tau_{i j k}$ and $\lambda_{i j k}$ denote the double stress and double couple stress tensors of gradient micropolar elasticity, respectively. We skip using the superscript ' $g$ ' for these components since the double (force and couple) stress tensors belong only to gradient version. These stress tensors are given by [29]

$$
\begin{aligned}
\sigma_{i j}^{g} & =\lambda \delta_{i j} \gamma_{k k}^{g}+(\mu+\eta) \gamma_{i j}^{g}+(\mu-\eta) \gamma_{j i}^{g}, \\
\mu_{i j}^{g} & =\alpha \delta_{i j} \kappa_{k k}^{g}+(\beta+\gamma) \kappa_{i j}^{g}+(\beta-\gamma) \kappa_{j i}^{g}, \\
\tau_{i j k} & =\epsilon^{2} \sigma_{i j, k}, \\
\lambda_{i j k} & =\epsilon^{2} \mu_{i j, k} .
\end{aligned}
$$

It is mentioned that instead of a general form of gradient micropolar elasticity, a specific simplified version is adopted. This version is closely connected to Eringen's nonlocal micropolar elasticity theory. As given in the constitutive relations (12), double stress and double couple stress tensors are simply the gradients of the stress and couple stress tensors, respectively, multiplied by $\epsilon^{2}$. Here, similar to nonlocal framework, $\epsilon$ is a characteristic material internal length. Moreover, $\gamma_{i j}^{g}$ and $\kappa_{i j}^{g}$ are the elastic micropolar distortion tensor and elastic micropolar bend-twist tensor within gradient micropolar elasticity, respectively, and are given by

$$
\begin{aligned}
\gamma_{i j}^{g} & =u_{i, j}^{g}+\epsilon_{i j k} \phi_{k}^{g}-\gamma_{i j}^{g, p}, \\
\kappa_{i j}^{g} & =\phi_{i, j}^{g}-\kappa_{i j}^{g, p} .
\end{aligned}
$$

Here, $u_{i}^{g}$ and $\phi_{i}^{g}$ denote the displacement and micro-rotation vectors of gradient micropolar elasticity, respectively, while $\gamma_{i j}^{g, p}$ and $\kappa_{i j}^{g, p}$ are the plastic micropolar distortion and plastic micropolar bend-twist tensors of gradient micropolar elasticity, respectively. It is noted that the elastic deformation measures of gradient theory (13) differ from the classical ones given by (6). In other words, the gradient micropolar elasticity includes deformation measures different from those of micropolar elasticity. This is in contrast to the case of nonlocal micropolar elasticity whose deformation measures are identical to those of micropolar elasticity, and no nonlocal deformation measures are defined.

\section{Variational formulation}

Once modeling defects such as cracks, traction boundary conditions are to be satisfied. Here, the relations provided in the previous sections are complemented with a variational formulation to determine the consistent (force and couple) traction boundary conditions. The variational formulation of classical micropolar elasticity is first reviewed [47], and then, the variational formulation of gradient micropolar elasticity is derived.

\subsection{Micropolar elasticity}

For an isotropic linear micropolar media, the strain energy density reads

$$
w^{c}=\frac{1}{2} \sigma_{i j}^{c} \gamma_{i j}^{c}+\frac{1}{2} \mu_{i j}^{c} \kappa_{i j}^{c} .
$$

The strain energy in a region $V$ occupied by the elastically deformed material is

$$
U^{c}=\int_{V} w^{c} \mathrm{~d} v=\frac{1}{2} \int_{V}\left(\sigma_{i j}^{c} \gamma_{i j}^{c}+\mu_{i j}^{c} \kappa_{i j}^{c}\right) \mathrm{d} v .
$$


The work done by external loads (including forces and couples) is given by

$$
W^{c}=\int_{V}\left(X_{i} u_{i}^{c}+Y_{i} \phi_{i}^{c}\right) \mathrm{d} v+\int_{A}\left(\bar{t}_{i} u_{i}^{c}+\bar{m}_{i} \phi_{i}^{c}\right) \mathrm{d} a
$$

where $X_{i}$ and $Y_{i}$ are body loads (i.e., body force and body couple, respectively) and $\bar{t}_{i}$ and $\bar{m}_{i}$ are the force and couple tractions, respectively. The total potential energy reads

$$
\Pi^{c}=U^{c}-W^{c}=\frac{1}{2} \int_{V}\left(\sigma_{i j}^{c} \gamma_{i j}^{c}+\frac{1}{2} \mu_{i j}^{c} \kappa_{i j}^{c}\right) \mathrm{d} v-\int_{V}\left(X_{i} u_{i}^{c}+Y_{i} \phi_{i}^{c}\right) \mathrm{d} v-\int_{A}\left(\bar{t}_{i} u_{i}^{c}+\bar{m}_{i} \phi_{i}^{c}\right) \mathrm{d} a
$$

Taking the first variation of $\Pi^{c}$ and considering the principle of minimum total potential energy results in

$$
\delta \Pi^{c}=\int_{V}\left(\sigma_{i j}^{c} \delta \gamma_{i j}^{c}+\mu_{i j}^{c} \delta \kappa_{i j}^{c}\right) \mathrm{d} v-\int_{V}\left(X_{i} \delta u_{i}^{c}+Y_{i} \delta \phi_{i}^{c}\right) \mathrm{d} v-\int_{A}\left(\bar{t}_{i} \delta u_{i}^{c}+\bar{m}_{i} \delta \phi_{i}^{c}\right) \mathrm{d} a=0 .
$$

Removing the plastic components, the variations in (elastic) micropolar distortion and (elastic) micropolar bend-twist tensors given in Eq. (6) read

$$
\begin{aligned}
& \delta \gamma_{i j}^{c}=\delta u_{i, j}^{c}+\epsilon_{i j k} \delta \phi_{k}^{c}, \\
& \delta \kappa_{i j}^{c}=\delta \phi_{i, j}^{c} .
\end{aligned}
$$

Hence, the principle of minimum total potential energy (18), after Gauss transformation and simplifying for smooth boundaries, leads to

$$
\begin{aligned}
\delta \Pi^{c}= & -\int_{V}\left(\sigma_{i j, j}^{c}+X_{i}\right) \delta u_{i}^{c} \mathrm{~d} v-\int_{V}\left(\mu_{i j, j}^{c}-\epsilon_{i j k} \sigma_{j k}^{c}+Y_{i}\right) \delta \phi_{i}^{c} \mathrm{~d} v \\
& +\int_{A}\left(\sigma_{i j}^{c} n_{j}-\bar{t}_{i}\right) \delta u_{i}^{c} \mathrm{~d} a+\int_{A}\left(\mu_{i j}^{c} n_{j}-\bar{m}_{i}\right) \delta \phi_{i}^{c} \mathrm{~d} a=0
\end{aligned}
$$

while $n_{j}$ is the normal vector to the surface. Accordingly, the equilibrium equations (1) in the presence of external body loads are obtained

$$
\begin{aligned}
& \sigma_{i j, j}^{c}+X_{i}=0, \\
& \mu_{i j, j}^{c}-\epsilon_{i j k} \sigma_{j k}^{c}+Y_{i}=0 .
\end{aligned}
$$

Moreover, six traction (natural) boundary conditions of micropolar elasticity read

$$
\begin{aligned}
& t_{i}^{c}=\bar{t}_{i}, \\
& m_{i}^{c}=\bar{m}_{i},
\end{aligned}
$$

where the force traction (or stress vector) $t_{i}^{c}$ and couple traction (or couple stress vector) $m_{i}^{c}$, which appear on a free surface, are defined as

$$
\begin{aligned}
& t_{i}^{c}=\sigma_{i j}^{c} n_{j}, \\
& m_{i}^{c}=\mu_{i j}^{c} n_{j} .
\end{aligned}
$$

Due to our definitions in the constitutive equations (2) and kinematic relations (5), the tractions are the stress tensors postmultiplied by normal vector. There are other forms of kinematic relations in the literature which would result in different forms of tractions (i.e., being the stress tensors premultiplied by normal vector).

\subsection{Nonlocal micropolar elasticity}

The natural boundary conditions within nonlocal micropolar elasticity are similar to those in micropolar elasticity and read

$$
\begin{aligned}
& t_{i}^{n}=\bar{t}_{i}, \\
& m_{i}^{n}=\bar{m}_{i} .
\end{aligned}
$$

The tractions $t_{i}^{n}$ and $m_{i}^{n}$ are similarly given by

$$
\begin{aligned}
& t_{i}^{n}=\sigma_{i j}^{n} n_{j}, \\
& m_{i}^{n}=\mu_{i j}^{n} n_{j} .
\end{aligned}
$$




\subsection{Gradient micropolar elasticity}

Gradient micropolar elasticity generalizes the classical continuum addressing both higher gradient and higher order. In one hand, by increasing the order of theory, the force traction is accompanied by couple tractions (as was the case in micropolar elasticity). On the other hand, a higher gradient formulation would lead to generalized force and couple tractions. Accordingly, within gradient micropolar elasticity, force and couple tractions are accompanied by double (or hyper) force and double (or hyper) couple tractions.

Here, a variational formulation is followed to derive these generalized traction vectors. The strain energy density of an isotropic linear gradient micropolar media is given by [29]

$$
w^{g}=\frac{1}{2} \sigma_{i j}^{g} \gamma_{i j}^{g}+\frac{1}{2} \mu_{i j}^{g} \kappa_{i j}^{g}+\frac{1}{2} \tau_{i j k} \gamma_{i j, k}^{g}+\frac{1}{2} \lambda_{i j k} \kappa_{i j, k}^{g} .
$$

For an elastically deformed material occupying the region $V$, the strain energy takes the form

$$
U^{g}=\int_{V} w^{g} \mathrm{~d} v=\frac{1}{2} \int_{V}\left(\sigma_{i j}^{g} \gamma_{i j}^{g}+\mu_{i j}^{g} \kappa_{i j}^{g}+\tau_{i j k} \gamma_{i j, k}^{g}+\lambda_{i j k} \kappa_{i j, k}^{g}\right) \mathrm{d} v .
$$

The work done by external loads (including forces and couples) is given by

$$
W^{g}=\int_{V}\left(X_{i} u_{i}^{g}+Y_{i} \phi_{i}^{g}\right) \mathrm{d} v+\int_{A}\left(\bar{t}_{i} u_{i}^{g}+\bar{q}_{i} D u_{i}^{g}+\bar{m}_{i} \phi_{i}^{g}+\bar{p}_{i} D \phi_{i}^{g}\right) \mathrm{d} a
$$

where $X_{i}$ and $Y_{i}$ are body force and couple, respectively, $\bar{t}_{i}$ and $\bar{m}_{i}$ are the force traction and couple traction, and $\bar{q}_{i}$ and $\bar{p}_{i}$ are the double force traction and double couple traction, respectively. Moreover, $D()$ is the differential operator for the normal (directional) derivative given by

$$
D u_{i}^{g}=n_{l} u_{i, l}^{g}, D \phi_{i}^{g}=n_{l} \phi_{i, l}^{g} .
$$

The total potential energy takes the form

$$
\begin{aligned}
\Pi^{g}= & U^{g}-W^{g}=\frac{1}{2} \int_{V}\left(\sigma_{i j}^{g} \gamma_{i j}^{g}+\mu_{i j}^{g} \kappa_{i j}^{g}+\tau_{i j k} \gamma_{i j, k}^{g}+\lambda_{i j k} \kappa_{i j, k}^{g}\right) \mathrm{d} v \\
& -\int_{V}\left(X_{i} u_{i}^{g}+Y_{i} \phi_{i}^{g}\right) \mathrm{d} v-\int_{A}\left(\bar{t}_{i} u_{i}^{g}+\bar{q}_{i} D u_{i}^{g}+\bar{m}_{i} \phi_{i}^{g}+\bar{p}_{i} D \phi_{i}^{g}\right) \mathrm{d} a .
\end{aligned}
$$

The principle of minimum total potential energy then leads to

$$
\begin{aligned}
\delta \Pi^{g}= & \int_{V}\left(\sigma_{i j}^{g} \delta \gamma_{i j}^{g}+\mu_{i j}^{g} \delta \kappa_{i j}^{g}+\tau_{i j k} \delta \gamma_{i j, k}^{g}+\lambda_{i j k} \delta \kappa_{i j, k}^{g}\right) \mathrm{d} v \\
& -\int_{V}\left(X_{i} \delta u_{i}^{g}+Y_{i} \delta \phi_{i}^{g}\right) \mathrm{d} v-\int_{A}\left(\bar{t}_{i} \delta u_{i}^{g}+\bar{q}_{i} D\left(\delta u_{i}^{g}\right)+\bar{m}_{i} \delta \phi_{i}^{g}+\bar{p}_{i} D\left(\delta \phi_{i}^{g}\right)\right) \mathrm{d} a=0 .
\end{aligned}
$$

Removing the plastic components, the variations of (elastic) micropolar distortion and (elastic) micropolar bend-twist tensors given in Eq. (13) read

$$
\begin{aligned}
\delta \gamma_{i j}^{g} & =\delta u_{i, j}^{g}+\epsilon_{i j k} \delta \phi_{k}^{g}, \\
\delta \kappa_{i j}^{g} & =\delta \phi_{i, j}^{g} .
\end{aligned}
$$

Hence, the principle of minimum total potential energy (31), after Gauss transformation and assuming smooth boundaries, leads to

$$
\begin{aligned}
\delta & \Pi^{g}=-\int_{V}\left(\left(\sigma_{i j}^{g}-\tau_{i j k, k}\right)_{, j}+X_{i}\right) \delta u_{i}^{g} \mathrm{~d} v-\int_{V}\left(\left(\mu_{i j}^{g}-\lambda_{i j k, k}\right)_{, j}-\epsilon_{i j k}\left(\sigma_{j k}^{g}-\tau_{j k l, l}\right)+Y_{i}\right) \delta \phi_{i}^{g} \mathrm{~d} v \\
& +\int_{A}\left(\left(\sigma_{i j}^{g}-\tau_{i j k, k}\right) n_{j}-\left(\tau_{i j k} n_{k}\right)_{, j}+\left(\tau_{i j k} n_{k} n_{l}\right)_{, l} n_{j}-\bar{t}_{i}\right) \delta u_{i}^{g} \mathrm{~d} a \\
& +\int_{A}\left(\left(\mu_{i j}^{g}-\lambda_{i j k, k}\right) n_{j}-\left(\lambda_{i j k}\right)_{, j} n_{k}+\left(\lambda_{i j k} n_{k} n_{l}\right)_{, l} n_{j}+\epsilon_{i l k} \tau_{l k j} n_{j}-\bar{m}_{i}\right) \delta \phi_{i}^{g} \mathrm{~d} a
\end{aligned}
$$




$$
+\int_{A}\left(\tau_{i j k} n_{j} n_{k}-\bar{q}_{i}\right) D\left(\delta u_{i}^{g}\right) \mathrm{d} a+\int_{A}\left(\lambda_{i j k} n_{j} n_{k}-\bar{p}_{i}\right) D\left(\delta \phi_{i}^{g}\right) \mathrm{d} a=0
$$

Consequently, the equilibrium equations (11) in the presence of external body loads are obtained

$$
\begin{aligned}
& \left(\sigma_{i j}^{g}-\partial_{k} \tau_{i j k}\right), j+X_{i}=0, \\
& \left(\mu_{i j}^{g}-\partial_{k} \lambda_{i j k}\right)_{, j}-\epsilon_{i j k}\left(\sigma_{j k}^{g}-\tau_{j k l, l}\right)+Y_{i}=0,
\end{aligned}
$$

while the traction boundary conditions of gradient micropolar elasticity take the form

$$
\begin{aligned}
t_{i}^{g} & =\bar{t}_{i}, \\
m_{i}^{g} & =\bar{m}_{i}, \\
q_{i} & =\bar{q}_{i}, \\
p_{i} & =\bar{p}_{i} .
\end{aligned}
$$

The force and couple tractions, and double force and double couple tractions are given by

$$
\begin{aligned}
& t_{i}^{g}=\left(\sigma_{i j}^{g}-\tau_{i j k, k}\right) n_{j}-\left(\tau_{i j k} n_{k}\right)_{, j}+\left(\tau_{i j k} n_{k} n_{l}\right)_{, l} n_{j} \\
& m_{i}^{g}=\left(\mu_{i j}^{g}-\lambda_{i j k, k}\right) n_{j}-\left(\lambda_{i j k} n_{k}\right)_{, j}+\left(\lambda_{i j k} n_{k} n_{l}\right)_{, l} n_{j}+\epsilon_{i l k} \tau_{l k j} n_{j}, \\
& q_{i}=\tau_{i j k} n_{j} n_{k} \\
& p_{i}=\lambda_{i j k} n_{j} n_{k}
\end{aligned}
$$

where the double stress tensor is appeared in the expression for couple traction.

\section{Line defects: dislocations and disclinations}

In the continuum theory of defects, the line defects being dislocation and disclination are the source of the plastic deformation measures, giving rise to an incompatible elasticity formulation. The dislocation (i.e., displacement discontinuity) breaks the translational symmetry, while with a disclination (i.e., rotational discontinuity), the rotational symmetry is violated. The dislocation density and disclination density tensors read

$$
\begin{aligned}
& \alpha_{i j}^{q}=\epsilon_{j k l}\left(\gamma_{i l, k}^{q}+\epsilon_{i k m} \kappa_{m l}^{q}\right)=-\epsilon_{j k l}\left(\gamma_{i l, k}^{q, p}+\epsilon_{i k m} \kappa_{m l}^{q, p}\right), \\
& \theta_{i j}^{q}=\epsilon_{j k l} \kappa_{i l, k}^{q}=-\epsilon_{j k l} \kappa_{i l, k}^{q, p},
\end{aligned}
$$

while $q \in\{c, n, g\}$ refers to classical micropolar, nonlocal micropolar, or gradient micropolar elasticity.

We consider dislocations and disclinations whose line are along $z$-axis of the Cartesian coordinate system. In this paper, we study plane crack problems. For this purpose, line defects with plane strain (i.e., edge dislocations and wedge disclinations) are reviewed in the following sections. It is noted that the generalized plane strain problems of micropolar elasticity are consisted of plane strain and antiplane strain cases. For isotropic micropolar elasticity, plane strain and antiplane strain cases are independent. For plane strain case, the displacement and micro-rotation vectors take the form $\left(u_{x}, u_{y}, 0\right)$ and $\left(0,0, \phi_{z}\right)$ giving rise to nonzero inplane stress components $\sigma_{x x}, \sigma_{x y}, \sigma_{y x}$, and $\sigma_{y y}$ and antiplane couple stress components $\mu_{z x}, \mu_{x z}, \mu_{y z}$, and $\mu_{z y}$.

In this section, we review the singular solutions to a glide edge dislocation [47] and a wedge disclination within micropolar elasticity. We also review the nonsingular solutions of a glide edge dislocation and a wedge disclination given by Lazar and Maugin [30] in the framework of gradient micropolar elasticity and nonlocal micropolar elasticity. Moreover, the singular and nonsingular solutions of a climb edge dislocation are derived here following a similar approach. In this paper, we do not investigate the energy of the line defects [35]. 


\subsection{Edge dislocation}

Consider now a straight edge dislocation whose line coincides with the $z$-axis. For a glide edge dislocation, the Burgers vector $b_{x}$ is parallel to the $x$-axis, while for a climb edge dislocation, the Burgers vector $b_{y}$ is parallel to the $y$-axis. Note that the Burgers vector has the dimension of length.

The stress functions for glide edge dislocation is derived by Nowacki [47] for micropolar elasticity and then generalized for gradient and nonlocal elasticity by Lazar and Maugin [30]. The stress functions of a climb edge dislocation are derived here following [47] for micropolar elasticity, and following [30,31] for nonlocal and gradient micropolar elasticity.

Within micropolar, nonlocal micropolar, and gradient micropolar elasticity, the stress field of these edge dislocations is given by [30]

$$
\begin{aligned}
\sigma_{x x}^{q} & =f_{, y y}^{q}-\psi_{, x y}^{q}, \\
\sigma_{x y}^{q} & =-f_{, x y}^{q}+\psi_{, x x}^{q}, \\
\sigma_{y x}^{q} & =-f_{, x y}^{q}-\psi_{, y y}^{q}, \\
\sigma_{y y}^{q} & =f_{, x x}^{q}+\psi_{, x y}^{q}, \\
\sigma_{z z}^{q} & =v \Delta f^{q},
\end{aligned}
$$

and the couple stress field reads

$$
\begin{aligned}
& \left\{\mu_{z x}^{q}, \mu_{x z}^{q}\right\}=\left\{1, \frac{\beta-\gamma}{\beta+\gamma}\right\} \psi_{, x}^{q}, \\
& \left\{\mu_{z y}^{q}, \mu_{y z}^{q}\right\}=\left\{1, \frac{\beta-\gamma}{\beta+\gamma}\right\} \psi_{, y}^{q} .
\end{aligned}
$$

Here, the stress functions $f^{q}$ and $\psi^{q}$ are given by [30]

$$
\begin{aligned}
f^{c}= & -\frac{\mu b_{x}}{2 \pi(1-v)} y\{\ln (r)\}+\frac{\mu b_{y}}{2 \pi(1-v)} x\{\ln (r)\}, \\
f^{n}= & f^{g}=-\frac{\mu b_{x}}{2 \pi(1-v)} y\left\{\ln (r)+\frac{2}{r^{2} / \epsilon^{2}}\left(1-\frac{r}{\epsilon} K_{1}(r / \epsilon)\right)\right\} \\
& +\frac{\mu b_{y}}{2 \pi(1-v)} x\left\{\ln (r)+\frac{2}{r^{2} / \epsilon^{2}}\left(1-\frac{r}{\epsilon} K_{1}(r / \epsilon)\right)\right\} \\
\psi^{c}= & -\frac{(\beta+\gamma) b_{x}}{2 \pi} \partial_{x}\left\{\ln (r)+K_{0}\left(r / l_{1}\right)\right\}-\frac{(\beta+\gamma) b_{y}}{2 \pi} \partial_{y}\left\{\ln (r)+K_{0}\left(r / l_{1}\right)\right\}, \\
\psi^{n}= & \psi^{g}=-\frac{(\beta+\gamma) b_{x}}{2 \pi} \partial_{x}\left\{\ln (r)+\frac{1}{1-\epsilon^{2} / l_{1}^{2}} K_{0}\left(r / l_{1}\right)-\frac{1}{l_{1}^{2} / \epsilon^{2}-1} K_{0}(r / \epsilon)\right\} \\
& -\frac{(\beta+\gamma) b_{y}}{2 \pi} \partial_{y}\left\{\ln (r)+\frac{1}{1-\epsilon^{2} / l_{1}^{2}} K_{0}\left(r / l_{1}\right)-\frac{1}{l_{1}^{2} / \epsilon^{2}-1} K_{0}(r / \epsilon)\right\},
\end{aligned}
$$

while again $q \in\{c, n, g\}$ addresses classical micropolar, nonlocal micropolar, or gradient micropolar elasticity. The stress and couple stress tensors are singular at the dislocation core in micropolar elasticity. However, they are regularized within gradient and nonlocal micropolar elasticity.

The double stress components of gradient micropolar elasticity read

$$
\begin{aligned}
& \tau_{x x y}=\epsilon^{2} \sigma_{x x, y}^{g}, \quad \tau_{x x x}=-\tau_{x y y}=\epsilon^{2} \sigma_{x x, x}^{g}, \\
& \tau_{x y x}=\epsilon^{2} \sigma_{x y, x}^{g}, \quad \tau_{y x x}=-\tau_{y y y}=\epsilon^{2} \sigma_{y x, x}^{g}, \\
& \tau_{y x y}=\epsilon^{2} \sigma_{y x, y}^{g}, \quad \tau_{y y x}=\epsilon^{2} \sigma_{y y, x}^{g}, \\
& \tau_{z z x}=\epsilon^{2} \sigma_{z z, x}^{g}, \tau_{z z y}=\epsilon^{2} \sigma_{z z, y}^{g},
\end{aligned}
$$

while the double couple stress components are given by

$$
\lambda_{z x x}=\epsilon^{2} \mu_{z x, x}^{g}, \lambda_{z y y}=\epsilon^{2} \mu_{z y, y}^{g}, \lambda_{z x y}=\lambda_{z y x}=\epsilon^{2} \mu_{z x, y}^{g},
$$




$$
\lambda_{x z x}=\epsilon^{2} \mu_{x z, x}^{g}, \lambda_{y z y}=\epsilon^{2} \mu_{y z, y}^{g}, \lambda_{x z y}=\lambda_{y z x}=\epsilon^{2} \mu_{x z, y}^{g} .
$$

The double stress tensor is singular at the dislocation core, while the double couple stress is nonsingular. Within micropolar and nonlocal micropolar elasticity, the dislocation density of the edge dislocation reads

$$
\alpha_{m z}^{n}=\alpha_{m z}^{c}=b_{m} \delta(x) \delta(y)
$$

while within gradient micropolar elasticity, we have [30]

$$
\alpha_{m z}^{g}=\frac{b_{m}}{2 \pi} \frac{1}{\epsilon^{2}} K_{0}(r / \epsilon)
$$

where $m$ refers to $x, y$.

\subsection{Wedge disclination}

We consider a straight wedge disclination with Frank vector $\omega_{z}$ whose line and Frank vectors are parallel to the $z$-axis of a coordinate system. Note that the Frank vector is dimensionless. Within micropolar, nonlocal micropolar, and gradient micropolar elasticity, the nonzero stress components read [30]

$$
\begin{aligned}
\left\{\sigma_{x x}^{q}, \sigma_{y y}^{q}, \sigma_{z z}^{q}\right\} & =\left\{\partial_{y y}, \partial_{x x}, v \Delta\right\} f^{q}, \\
\sigma_{x y}^{q}=\sigma_{y x}^{q} & =-f_{, x y}^{q}
\end{aligned}
$$

and the couple stress field read

$$
\begin{aligned}
& \left\{\mu_{z x}^{q}, \mu_{x z}^{q}\right\}=-\left\{1, \frac{\beta-\gamma}{\beta+\gamma}\right\} \psi_{, y}^{q}, \\
& \left\{\mu_{z y}^{q}, \mu_{y z}^{q}\right\}=\left\{1, \frac{\beta-\gamma}{\beta+\gamma}\right\} \psi_{, x}^{q} .
\end{aligned}
$$

Here, the stress functions are given by [30]

$$
\begin{aligned}
f^{c} & =\frac{\mu \omega_{z}}{4 \pi(1-v)} r^{2}\left(\ln r-\frac{1}{2}\right), \\
f^{n} / b_{x} & =f^{g} / b_{x}=\frac{\mu \omega_{z}}{4 \pi(1-v)}\left\{r^{2}\left(\ln r-\frac{1}{2}\right)+4 \epsilon^{2}\left(\ln r+K_{0}(r / \epsilon)+\frac{1}{2}\right)\right\}, \\
\psi^{c} & =\frac{(\beta+\gamma) \omega_{z}}{2 \pi} \ln r, \\
\psi^{n} & =\psi^{g} / b_{x}=\frac{(\beta+\gamma) \omega_{z}}{2 \pi}\left(\ln r+K_{0}(r / \epsilon)\right) .
\end{aligned}
$$

Similar to edge dislocations, the stress and couple stress tensors of a wedge disclination are singular in micropolar elasticity. However, they are regularized within gradient and nonlocal micropolar elasticity.

The double stress components of gradient micropolar elasticity read

$$
\begin{aligned}
& \tau_{x x y}=\epsilon^{2} \sigma_{x x, y}^{g}, \quad \tau_{x x x}=-\tau_{x y y}=\epsilon^{2} \sigma_{x x, x}^{g}, \\
& \tau_{x y x}=\epsilon^{2} \sigma_{x y, x}^{g}, \quad \tau_{y x x}=-\tau_{y y y}=\epsilon^{2} \sigma_{y x, x}^{g}, \\
& \tau_{y x y}=\epsilon^{2} \sigma_{y x, y}^{g}, \quad \tau_{y y x}=\epsilon^{2} \sigma_{y y, x}^{g}, \\
& \tau_{z z x}=\epsilon^{2} \sigma_{z z, x}^{g}, \quad \tau_{z z y}=\epsilon^{2} \sigma_{z z, y}^{g},
\end{aligned}
$$

while the double couple stress components are given by

$$
\begin{array}{ll}
\lambda_{z x y}=\epsilon^{2} \mu_{z x, y}^{g}, & \lambda_{z y x}=\epsilon^{2} \mu_{z y, x}^{g},
\end{array}
$$


Interestingly, the double stress tensor is nonsingular at the dislocation core, while the double couple stress is singular.

Within micropolar and nonlocal micropolar elasticity, the disclination density of the wedge disclination reads

$$
\theta_{z z}^{n}=\theta_{z z}^{c}=\omega_{z} \delta(x) \delta(y)
$$

while within gradient micropolar elasticity we have [30]

$$
\theta_{z z}^{g}=\frac{\omega_{z}}{2 \pi} \frac{1}{\epsilon^{2}} K_{0}(r / \epsilon)
$$

\section{Dislocation- and disclination-based micropolar fracture mechanics}

The continuum mechanics theories are generalized to study features which are not visible to classical theories. In order to establish and employ a generalized continuum theory, different aspects of material behavior should be addressed. Fracture mechanics is among the subjects which are essential in our understanding of material behavior. In the previous sections, the frameworks including micropolar, nonlocal micropolar, and gradient micropolar elasticity are reviewed. A variational approach is also provided to formulate the traction boundary conditions. Additionally, the solutions to the discrete dislocations are also reviewed. These formulations can now serve as modules to model and study cracks within these continuum theories.

Dislocation-based fracture (or distributed dislocation technique) is a classical method to analyze cracks by representing them as a continuous distribution of dislocations. This technique has been successfully developed for classical elasticity and is recently employed within generalized continuum theories such as (Helmholtz and bi-Helmholtz type) nonlocal and gradient elasticity details of which are reported in the introduction.

Within micropolar elasticity, the material points possess six degrees of freedom including three displacement $\left(u_{x}, u_{y}, u_{z}\right)$ together with three micro-rotations $\left(\phi_{x}, \phi_{y}, \phi_{z}\right)$. Once a crack is studied within a micropolar media with such deformation measures, both discontinuities of displacement and micro-rotation should be taken into account. Accordingly, instead of the classical method of dislocation-based fracture, we shall use a dislocation- and disclination (i.e., in general a line defect)-based fracture mechanics for polar media. In other words, depending on the discontinuity of the deformation measures across the crack line, a consistent set of line defects (including screw and edge dislocations and wedge and twist disclinations) are to be selected. In the following, this selection is shown to be systematic by simply satisfying the corresponding boundary conditions. Enforcing the crack-face boundary conditions, the densities of the line defects are determined. We now use this technique to study inplane cracks within micropolar, nonlocal micropolar, and gradient micropolar elasticity.

\subsection{Cracks in micropolar elasticity}

Consider a plane weakened by one straight crack of length $2 a$ along $x$-axis (Fig. 2), parametrically represented as

$$
\begin{aligned}
& x=a s \quad-1 \leq s \leq 1, \\
& y=0 .
\end{aligned}
$$

Within micropolar elasticity, the force and couple tractions of a surface with normal vector $n_{i}$ are given by Eq. (23). The tractions on the surface of such planar crack (for which $n_{x}=n_{z}=0, n_{y}=1$, Fig. 2) are

$$
\begin{aligned}
t_{x}^{c} & =\sigma_{x y}^{c}, \quad t_{y}^{c}=\sigma_{y y}^{c}, \quad t_{z}^{c}=\sigma_{z y}^{c}, \\
m_{x}^{c} & =\mu_{x y}^{c}, \quad m_{y}^{c}=\mu_{y y}^{c}, \quad m_{z}^{c}=\mu_{z y}^{c} .
\end{aligned}
$$

Dyskin and Pasternak [11] have employed the symmetry conditions to select suitable topological defects to represent complex defects such as cracks in polar media. Here, we intend to develop another comprehensive approach for this task. A crack can affect the stress or couple stress field (and deformation and rotation fields) through its tractions. Accordingly, only remote loadings corresponding to the components given in (53) will sense the crack and induce deformation and/or micro-rotation, and thus, we need to study planes under corresponding remote loadings. These remote loadings can be categorized as plane strain and antiplane strain cases. 


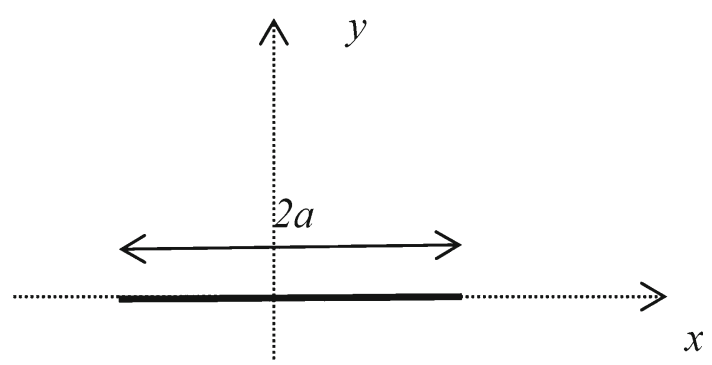

Fig. 2 Plane weakened by one crack

The remote loadings $\sigma_{x y}^{\infty}, \sigma_{y y}^{\infty}$, and $\mu_{z y}^{\infty}$ give rise to plane strain case with $u_{x}, u_{y}$, and $\phi_{z}$ fields. The loadings $\sigma_{z y}^{\infty}$, $\mu_{x y}^{\infty}$, and $\mu_{y y}^{\infty}$ give rise to antiplane strain case with $u_{z}, \phi_{x}$, and $\phi_{y}$ fields. In this study, we only address plane strain case where a distribution of edge dislocations and wedge disclination can successfully represent the crack. For the case of antiplane strain, which is left for future studies, the first impression is to employ screw dislocation and twist disclinations. However, it is to be noted that twist disclinations give rise to nonzero inplane stress as well as inplane displacement fields [49] being a linear functions of $z$ within classical micropolar elasticity [9,27].

Within plane strain micropolar elasticity, the plane (Fig. 2) is assumed to be subjected to the remote loadings

$$
\sigma_{x y}^{\infty}=\sigma_{x y_{0}}, \quad \sigma_{y y}^{\infty}=\sigma_{y y_{0}}, \quad \mu_{z y}^{\infty}=\mu_{z y_{0}} .
$$

Note that in micropolar (asymmetric) elasticity, the stress and couple stress tensors are not symmetric, and for the stress tensor $\sigma_{i j}^{c}$ and couple stress tensor $\mu_{i j}^{c}$, the second subscript identifies the area element, whereas the first subscript shows the direction of the stress action (see Fig. 1).

Considering Bueckner's superposition principle [5], the crack problem is decomposed into two subproblems. In one hand, the first subproblem deals with an uncracked body under remote normal loading (54). In this case, the solution to the micropolar elasticity problem leads to

$$
\sigma_{x y}^{c}=\sigma_{x y_{0}}, \quad \sigma_{y y}^{c}=\sigma_{y y_{0}}, \quad \mu_{z y}^{c}=\mu_{z y_{0}}
$$

for subproblem one while all other stress components as well as couple stress components vanish. Accordingly, the tractions along the (nonexisting) crack surface read

$$
\begin{aligned}
& \bar{t}_{x}=\sigma_{x y_{0}}, \quad \bar{t}_{y}=\sigma_{y y_{0}}, \quad \bar{t}_{z}=0, \\
& \bar{m}_{x}=\bar{m}_{y}=0, \quad \bar{m}_{z}=\mu_{z y_{0}} .
\end{aligned}
$$

The second subproblem is an uncracked body in the absence of remote loading but subjected to equal and opposite tractions present along the line of the crack in the first subproblem (56), i.e.,

$$
\begin{aligned}
& \bar{t}_{x}=-\sigma_{x y_{0}}, \quad \bar{t}_{y}=-\sigma_{y y_{0}}, \quad \bar{t}_{z}=0, \\
& \bar{m}_{x}=\bar{m}_{y}=0, \quad \bar{m}_{z}=-\mu_{z y_{0}},
\end{aligned}
$$

with vanishing far-field stress and couple stress components.

In the second subproblem, the tractions (57) can be produced by a distribution of line defects (i.e., dislocations and/or disclinations). As stated earlier, edge dislocation and wedge disclination correspond to plane strain case. Therefore, cracks in plane strain micropolar elasticity can be represented by distributions of edge dislocations and wedge disclinations. In other words, in this case the crack line may possess discontinuities of deformation $u_{x}$ and $u_{y}$ and micro-rotation $\phi_{z}$. This implies that the edge dislocation (i.e., discontinuity of displacement $u_{x}$ and $u_{y}$ ) and wedge disclination (i.e., discontinuity of micro-rotation $\phi_{z}$ ) are consistent with the deformation and rotation fields. The density of these line defects is to be determined using the integral equations obtained based on the crack surface conditions. Then, the first and second subproblem can be superposed to provide the complete solution of the original problem.

Substituting the tractions (57) and the expressions (53) into the traction boundary conditions (22), we have

$$
\begin{aligned}
& \sigma_{x y}^{c}=-\sigma_{x y 0}, \quad \sigma_{y y}^{c}=-\sigma_{y y 0}, \quad \sigma_{z y}^{c}=0, \\
& \mu_{x y}^{c}=\mu_{y y}^{c}=0, \quad \mu_{z y}^{c}=-\mu_{z y 0},
\end{aligned}
$$


along the line of the crack, i.e., $-a \leq x \leq a, y=0$.

Considering the crack as a distribution of climb and glide edge dislocations and wedge disclinations, we are left with the tractions for plane strain cases, i.e.,

$$
\sigma_{x y}^{c}=-\sigma_{x y 0}, \quad \sigma_{y y}^{c}=-\sigma_{y y 0}, \quad \mu_{z y}^{c}=-\mu_{z y 0} .
$$

For these line defects, the stress and couple stress components are given in (38), (39), (45), and (46). Considering these components, the traction boundary conditions (59) lead to the following integral equations for the density of the line defects

$$
\begin{aligned}
& \int_{-1}^{1} K_{11}^{c}(s, \xi) B_{x}^{c}(\xi) \mathrm{d} \xi=-\sigma_{x y_{0}} \\
& \int_{-1}^{1}\left\{K_{22}^{c}(s, \xi) B_{y}^{c}(\xi)+K_{23}^{c}(s, \xi) \Omega_{z}^{c}(\xi)\right\} \mathrm{d} \xi=-\sigma_{y y_{0}} \\
& \int_{-1}^{1}\left\{K_{32}^{c}(s, \xi) B_{y}^{c}(\xi)+K_{33}^{c}(s, \xi) \Omega_{z}^{c}(\xi)\right\} \mathrm{d} \xi=-\mu_{z y_{0}}
\end{aligned}
$$

while the kernels $K_{i j}^{c}(s, \xi)$ are deduced from the field components of the corresponding discrete line defects provided in the previous section. Moreover, $B_{x}^{c}, B_{y}^{c}$, and $\Omega_{z}^{c}$ are the densities of the glide edge dislocation, climb edge dislocation, and wedge disclination, respectively, within the (classical) micropolar elasticity. It is noted that the traction conditions $\sigma_{z y}^{c}=0$ and $\mu_{x y}^{c}=\mu_{y y}^{c}=0$ in (58) are automatically satisfied since the corresponding stress and couple stress components vanish for edge dislocations and wedge disclinations. Furthermore, along $y=0, \sigma_{x y}=0$ for climb edge dislocation and wedge disclination while $\sigma_{y y}=0$, and $\mu_{z y}=0$ for glide dislocation.

The closure requirements (the condition for single-valuedness) of displacement and rotation outside the crack are given by

$$
\int_{-1}^{1} B_{x}^{c}(\xi) \mathrm{d} \xi=0, \quad \int_{-1}^{1} B_{y}^{c}(\xi) \mathrm{d} \xi=0, \quad \int_{-1}^{1} \Omega_{z}^{c}(\xi) \mathrm{d} \xi=0 .
$$

The solution to the system of integral equations (60) and (61) gives the density of the dislocations and disclination (i.e., $B_{x}^{c}, B_{y}^{c}$, and $\Omega_{z}^{c}$ ) which represents the crack. These densities can be used to determine the stress and couple stress tensor in the second subproblem.

The superposition of the first and second subproblems is equivalent to the original problem, i.e., a plane weakened by a crack under loading (54). Accordingly, the stress and couple stress fields in the original problem is the superposition of those fields in the subproblems. In particular, we have

$$
\begin{aligned}
& \sigma_{x y}^{c}(x, y)=\sigma_{x y_{0}}+\int_{-1}^{1}\left\{S_{1}^{c} B_{x}^{c}(\xi)+S_{2}^{c} B_{y}^{c}(\xi)+S_{3}^{c} \Omega_{z}^{c}(\xi)\right\} \mathrm{d} \xi \\
& \sigma_{y y}^{c}(x, y)=\sigma_{y y_{0}}+\int_{-1}^{1}\left\{T_{1}^{c} B_{x}^{c}(\xi)+T_{2}^{c} B_{y}^{c}(\xi)+T_{3}^{c} \Omega_{z}^{c}(\xi)\right\} \mathrm{d} \xi \\
& \mu_{z y}^{c}(x, y)=\mu_{z y_{0}}+\int_{-1}^{1}\left\{M_{1}^{c} B_{x}^{c}(\xi)+M_{2}^{c} B_{y}^{c}(\xi)+M_{3}^{c} \Omega_{z}^{c}(\xi)\right\} \mathrm{d} \xi
\end{aligned}
$$

The kernels $S_{i}, T_{i}$, and $M_{i}$ are deduced from the field components of the corresponding discrete line defects.

\subsection{Cracks in nonlocal micropolar elasticity}

We have studied so far the analysis of a crack within micropolar elasticity. Here, the analysis will be extended to the nonlocal micropolar elasticity. Consider again a plane weakened by one straight crack of length $2 a$ along $x$-axis (Fig. 2), parametrically represented as (52).

Within nonlocal micropolar elasticity, the force and couple tractions of a surface with normal vector $n_{i}$ are given by Eq. (25). Accordingly, the tractions on the surface of the crack (for which $n_{x}=n_{z}=0, n_{y}=1$ ) are

$$
t_{x}^{n}=\sigma_{x y}^{n}, \quad t_{y}^{n}=\sigma_{y y}^{n}, \quad t_{z}^{n}=\sigma_{z y}^{n},
$$




$$
m_{x}^{n}=\mu_{x y}^{n}, \quad m_{y}^{n}=\mu_{y y}^{n}, \quad m_{z}^{n}=\mu_{z y}^{n} .
$$

Consider the plane (Fig. 2) under remote normal loading (54). Similar to the previous section, the original problem is divided here into two subproblems.

The first subproblem deals with an uncracked body under remote normal loading (54). In this case, the solution to the micropolar elasticity problem leads to

$$
\sigma_{x y}^{n}=\sigma_{x y_{0}}, \quad \sigma_{y y}^{n}=\sigma_{y y_{0}}, \quad \mu_{z y}^{n}=\mu_{z y_{0}}
$$

while all other stress components as well as couple stress components vanish. Accordingly, the tractions along the (nonexisting) crack surface read

$$
\begin{aligned}
& \bar{t}_{x}=\sigma_{x y_{0}}, \quad \bar{t}_{y}=\sigma_{y y_{0}}, \quad \bar{t}_{z}=0, \\
& \bar{m}_{x}=\bar{m}_{y}=0, \quad \bar{m}_{z}=\mu_{z y_{0}} .
\end{aligned}
$$

The second subproblem is an uncracked body in the absence of remote loading but subjected to equal and opposite tractions present along the line of the crack in the first subproblem (65), i.e.,

$$
\begin{aligned}
& \bar{t}_{x}=-\sigma_{x y_{0}}, \quad \bar{t}_{y}=-\sigma_{y y_{0}}, \quad \bar{t}_{z}=0 \\
& \bar{m}_{x}=\bar{m}_{y}=0, \quad \bar{m}_{z}=-\mu_{z y_{0}},
\end{aligned}
$$

with vanishing far-field stress and couple stress components.

Substituting the tractions (66) and the expressions (63) into the traction boundary conditions (24), we have

$$
\begin{aligned}
& \sigma_{x y}^{n}=-\sigma_{x y 0}, \quad \sigma_{y y}^{n}=-\sigma_{y y 0}, \quad \sigma_{z y}^{n}=0, \\
& \mu_{x y}^{n}=\mu_{y y}^{n}=0, \quad \mu_{z y}^{n}=-\mu_{z y 0},
\end{aligned}
$$

along the line of the crack, i.e., $-a \leq x \leq a, y=0$.

Considering the crack as a distribution of climb and glide edge dislocations and wedge disclinations, we are left with the tractions for plane strain case, i.e.,

$$
\sigma_{x y}^{n}=-\sigma_{x y 0}, \quad \sigma_{y y}^{n}=-\sigma_{y y 0}, \quad \mu_{z y}^{n}=-\mu_{z y 0} .
$$

The stress and couple stress components given in (38), (39), (45), and (46), together with the traction boundary conditions (68) lead to the following integral equations for the density of the line defects

$$
\begin{aligned}
& \int_{-1}^{1} K_{11}^{n}(s, \xi) B_{x}^{n}(\xi) \mathrm{d} \xi=-\sigma_{x y_{0}} \\
& \int_{-1}^{1}\left\{K_{22}^{n}(s, \xi) B_{y}^{n}(\xi)+K_{23}^{n}(s, \xi) \Omega_{z}^{n}(\xi)\right\} \mathrm{d} \xi=-\sigma_{y y_{0}} \\
& \int_{-1}^{1}\left\{K_{32}^{n}(s, \xi) B_{y}^{n}(\xi)+K_{33}^{n}(s, \xi) \Omega_{z}^{n}(\xi)\right\} \mathrm{d} \xi=-\mu_{z y_{0}} .
\end{aligned}
$$

Similar to classical case, the kernels $K_{i j}^{n}(s, \xi)$ are deduced from the field components of the corresponding discrete line defects provided in the previous section. It is noted that the other traction conditions in (67) are automatically satisfied.

Furthermore, the closure requirements for displacement and rotation outside the crack are given by

$$
\int_{-1}^{1} B_{x}^{n}(\xi) \mathrm{d} \xi=0, \quad \int_{-1}^{1} B_{y}^{n}(\xi) \mathrm{d} \xi=0, \quad \int_{-1}^{1} \Omega_{z}^{n}(\xi) \mathrm{d} \xi=0 .
$$

The solution to the system of integral equations (69) and (70) gives the density of the line defects. The stress and couple stress fields in the original problem are the superposition of those fields in the subproblems. In particular, we have

$$
\sigma_{x y}^{n}(x, y)=\sigma_{x y_{0}}+\int_{-1}^{1}\left\{S_{1}^{n} B_{x}^{n}(\xi)+S_{2}^{n} B_{y}^{n}(\xi)+S_{3}^{n} \Omega_{z}^{n}(\xi)\right\} \mathrm{d} \xi
$$




$$
\begin{aligned}
& \sigma_{y y}^{n}(x, y)=\sigma_{y y_{0}}+\int_{-1}^{1}\left\{T_{1}^{n} B_{x}^{n}(\xi)+T_{2}^{n} B_{y}^{n}(\xi)+T_{3}^{n} \Omega_{z}^{n}(\xi)\right\} \mathrm{d} \xi \\
& \mu_{z y}^{n}(x, y)=\mu_{z y_{0}}+\int_{-1}^{1}\left\{M_{1}^{n} B_{x}^{n}(\xi)+M_{2}^{n} B_{y}^{n}(\xi)+M_{3}^{n} \Omega_{z}^{n}(\xi)\right\} \mathrm{d} \xi
\end{aligned}
$$

The kernels $S_{i}, T_{i}$, and $M_{i}$ are deduced from the field components of the corresponding discrete line defects.

\subsection{Cracks in gradient micropolar elasticity}

Consider again the plane weakened by one straight crack of length $2 a$ along $x$-axis (Fig. 2). Within gradient micropolar elasticity, the force and couple tractions of a surface with normal vector $n_{i}$ are given by Eq. (36). Simplifying the relations for the plane condition (no dependence on $z$ ), the tractions on the surface of the crack (for which $n_{x}=n_{z}=0, n_{y}=1$ ) are

$$
\begin{aligned}
t_{i}^{g} & =\sigma_{i y}^{g}-\epsilon^{2}\left(\sigma_{i y, \chi \chi}^{g}+\sigma_{i \chi, y \chi}^{g}-\sigma_{i y, y y}^{g}\right), \\
m_{i}^{g} & =\mu_{i y}^{g}-\epsilon^{2}\left(\mu_{i y, \chi \chi}^{g}+\mu_{i \chi, y \chi}^{g}-\mu_{i y, y y}^{g}\right)+\epsilon^{2} \epsilon_{i l k} \sigma_{l k, y}^{g}, \\
q_{i} & =\epsilon^{2} \sigma_{i y, y}^{g}, \\
p_{i} & =\epsilon^{2} \mu_{i y, y}^{g}
\end{aligned}
$$

while $\chi \in\{x, y\}$.

Consider again the plane (Fig. 2) under remote normal loading (54). Similar to the previous section, the original problem is divided into two subproblems. The first subproblem deals with an uncracked body under remote normal loading (54). In this case, the solution to the gradient micropolar elasticity problem leads to

$$
\sigma_{x y}^{g}=\sigma_{x y_{0}}, \quad \sigma_{y y}^{g}=\sigma_{y y_{0}}, \quad \mu_{z y}^{g}=\mu_{z y_{0}}
$$

while all other stress and double stress components as well as couple stress and double couple stress components vanish. Accordingly, the tractions along the (nonexisting) crack surface read

$$
\begin{aligned}
& \bar{t}_{x}=\sigma_{x y_{0}}, \quad \bar{t}_{y}=\sigma_{y y_{0}}, \quad \bar{t}_{z}=0, \\
& \bar{m}_{x}=\bar{m}_{y}=0, \quad \bar{m}_{z}=\mu_{z y_{0}}, \\
& \bar{q}_{x}=\bar{q}_{y}=\bar{q}_{z}=0, \\
& \bar{p}_{x}=\bar{p}_{y}=\bar{p}_{z}=0 .
\end{aligned}
$$

The second subproblem is an uncracked body in the absence of remote loading, but subjected to equal and opposite tractions present along the line of the crack in the first subproblem (74), i.e.,

$$
\begin{aligned}
& \bar{t}_{x}=-\sigma_{x y_{0}}, \quad \bar{t}_{y}=-\sigma_{y y_{0}}, \quad \bar{t}_{z}=0, \\
& \bar{m}_{x}=\bar{m}_{y}=0, \quad \bar{m}_{z}=-\mu_{z y_{0}}, \\
& \bar{q}_{x}=\bar{q}_{y}=\bar{q}_{z}=0 \\
& \bar{p}_{x}=\bar{p}_{y}=\bar{p}_{z}=0
\end{aligned}
$$

with vanishing far-field stress, double stress, couple stress, and double couple stress components.

Substituting the tractions (75) and the expressions (72) into the traction boundary conditions (35), we have

$$
\begin{aligned}
& \sigma_{x y}^{g}-\epsilon^{2}\left(\sigma_{x y, x x}^{g}+\sigma_{x y, y y}^{g}+\sigma_{x x, x y}^{g}\right)=-\sigma_{x y 0}, \\
& \sigma_{y y}^{g}-\epsilon^{2}\left(\sigma_{y y, x x}^{g}+\sigma_{y y, y y}^{g}+\sigma_{y x, x y}^{g}\right)=-\sigma_{y y 0}, \\
& \sigma_{z y}^{g}-\epsilon^{2}\left(\sigma_{z y, x x}^{g}+\sigma_{z y, y y}^{g}+\sigma_{z x, x y}^{g}\right)=0, \\
& \mu_{x y}^{g}-\epsilon^{2}\left(\mu_{x y, x x}^{g}+\mu_{x y, y y}^{g}+\mu_{x x, x y}^{g}\right)+\epsilon^{2} \sigma_{y z, y}^{g}-\epsilon^{2} \sigma_{z y, y}^{g}=0, \\
& \mu_{y y}^{g}-\epsilon^{2}\left(\mu_{y y, x x}^{g}+\mu_{y y, y y}^{g}+\mu_{y x, x y}^{g}\right)+\epsilon^{2} \sigma_{z x, y}^{g}-\epsilon^{2} \sigma_{x z, y}^{g}=0, \\
& \mu_{z y}^{g}-\epsilon^{2}\left(\mu_{z y, x x}^{g}+\mu_{z y, y y}^{g}+\mu_{z x, x y}^{g}\right)+\epsilon^{2} \sigma_{x y, y}^{g}-\epsilon^{2} \sigma_{y x, y}^{g}=-\mu_{z y 0},
\end{aligned}
$$




$$
\begin{aligned}
& \epsilon^{2} \sigma_{x y, y}^{g}=\epsilon^{2} \sigma_{y y, y}^{g}=\epsilon^{2} \sigma_{z y, y}^{g}=0, \\
& \epsilon^{2} \mu_{x y, y}^{g}=\epsilon^{2} \mu_{y y, y}^{g}=\epsilon^{2} \mu_{z y, y}^{g}=0,
\end{aligned}
$$

along the line of the crack, i.e., $-a \leq x \leq a, y=0$.

We now consider the crack as a distribution of climb and glide edge dislocations and wedge disclinations. Being a plane strain case, the only nonzero stress components are $\sigma_{x x}, \sigma_{x y}, \sigma_{y x}$, and $\sigma_{y y}$ and nonzero couple stress components are $\mu_{z x}, \mu_{x z}, \mu_{y z}$, and $\mu_{z y}$. Accordingly, the traction boundary conditions (76) are reduced to

$$
\begin{aligned}
& \sigma_{x y}^{g}-\epsilon^{2}\left(\sigma_{x y, x x}^{g}+\sigma_{x y, y y}^{g}+\sigma_{x x, x y}^{g}\right)=-\sigma_{x y 0}, \\
& \sigma_{y y}^{g}-\epsilon^{2}\left(\sigma_{y y, x x}^{g}+\sigma_{y y, y y}^{g}+\sigma_{y x, x y}^{g}\right)=-\sigma_{y y 0}, \\
& \mu_{z y}^{g}-\epsilon^{2}\left(\mu_{z y, x x}^{g}+\mu_{z y, y y}^{g}+\mu_{z x, x y}^{g}\right)+\epsilon^{2} \sigma_{x y, y}^{g}-\epsilon^{2} \sigma_{y x, y}^{g}=-\mu_{z y 0}, \\
& \epsilon^{2} \sigma_{x y, y}^{g}=\epsilon^{2} \sigma_{y y, y}^{g}=0, \\
& \epsilon^{2} \mu_{z y, y}^{g}=0,
\end{aligned}
$$

along the line of the crack.

In this step, we aim for an approximate solution for which we ignore the boundary conditions on double force tractions (77d) and double couple traction (77e). For the climb and glide edge dislocations and wedge disclinations, the stress and couple stress components given in (38), (39), (45), and (46), together with the traction boundary conditions (77) (excluding those of double tractions) lead to the following integral equations for the density of the line defects

$$
\begin{aligned}
& \int_{-1}^{1}\left\{K_{11}^{g}(s, \xi) B_{x}^{g}(\xi)+K_{12}^{g}(s, \xi) B_{y}^{g}(\xi)+K_{13}^{g}(s, \xi) \Omega_{z}^{g}(\xi)\right\} \mathrm{d} \xi=-\sigma_{x y_{0}} \\
& \int_{-1}^{1}\left\{K_{21}^{g}(s, \xi) B_{x}^{g}(\xi)+K_{22}^{g}(s, \xi) B_{y}^{g}(\xi)+K_{23}^{g}(s, \xi) \Omega_{z}^{n}(\xi)\right\} \mathrm{d} \xi=-\sigma_{y y_{0}} \\
& \int_{-1}^{1}\left\{K_{31}^{g}(s, \xi) B_{x}^{g}(\xi)+K_{32}^{g}(s, \xi) B_{y}^{g}(\xi)+K_{33}^{g}(s, \xi) \Omega_{z}^{n}(\xi)\right\} \mathrm{d} \xi=-\mu_{z y_{0}}
\end{aligned}
$$

while the kernels $K_{i j}^{g}(s, \xi)$ can be derived by substituting the discrete defect fields into Eq. (77). Furthermore, the closure requirements are given by

$$
\int_{-1}^{1} B_{x}^{g}(\xi) \mathrm{d} \xi=0, \quad \int_{-1}^{1} B_{y}^{g}(\xi) \mathrm{d} \xi=0, \quad \int_{-1}^{1} \Omega_{z}^{g}(\xi) \mathrm{d} \xi=0
$$

The solution to the system of integral equations (78) and (79) gives the density of the dislocations and disclination. The stress and couple stress fields in the original problem are the superposition of those fields in the subproblems. In particular, we have

$$
\begin{aligned}
& \sigma_{x y}^{g}(x, y)=\sigma_{x y_{0}}+\int_{-1}^{1}\left\{S_{1}^{g} B_{x}^{g}(\xi)+S_{2}^{g} B_{y}^{g}(\xi)+S_{3}^{g} \Omega_{z}^{g}(\xi)\right\} \mathrm{d} \xi \\
& \sigma_{y y}^{g}(x, y)=\sigma_{y y_{0}}+\int_{-1}^{1}\left\{T_{1}^{g} B_{x}^{g}(\xi)+T_{2}^{g} B_{y}^{g}(\xi)+T_{3}^{g} \Omega_{z}^{g}(\xi)\right\} \mathrm{d} \xi \\
& \mu_{z y}^{g}(x, y)=\mu_{z y_{0}}+\int_{-1}^{1}\left\{M_{1}^{g} B_{x}^{g}(\xi)+M_{2}^{g} B_{y}^{g}(\xi)+M_{3}^{g} \Omega_{z}^{g}(\xi)\right\} \mathrm{d} \xi
\end{aligned}
$$

The kernels are deduced from the field components of the corresponding discrete line defects. 


\section{Numerical results}

In the previous section, the general loading case of plane strain is considered and the formulation for cracks undergoing plane strain is presented. In this section, specific loadings are discussed as examples. For solving the integral equations, the method of singular-value decomposition is used considering a nonuniform distribution of 200 integration points (denser at the edges). We assume $\sigma_{x y_{0}}=\sigma_{y y_{0}}=\mu$ and $\mu_{z y 0}=\beta / l_{1}$. As the micropolar media, we consider Polystyrene foam with properties $\mu=0.607 e 6 \mathrm{MPa}, \eta=0.060 e 6 \mathrm{MPa}$, $\beta=8.77 \mathrm{~N}, \gamma=52 \mathrm{~N}, \alpha=-5.84 \mathrm{~N}, \lambda=0.099 e 6 \mathrm{MPa}$, and $v=0.07[20,26]$.

\subsection{Case 1}

As the first example, the crack is considered to be under remote loading $\sigma_{y y}^{\infty}=\sigma_{y y_{0}}$ while $\sigma_{x y}^{\infty}=0$ and $\mu_{z y}^{\infty}=0$. Considering the governing integral equations, in this case, a distribution of climb edge dislocation and wedge disclination are needed to represent the crack. For micropolar elasticity, having solved the governing integral equations (60) together with the closure conditions (61), the density of the climb edge dislocation and wedge disclination are calculated. Similarly, for nonlocal micropolar elasticity, the integral equations (69) and closure conditions (70) give the densities. Finally, the solution to the integral equations (78) together with closure conditions (79) leads to the densities in the gradient micropolar elasticity.

Figures 3 and 4 depict the densities of the climb edge dislocations $\left(B_{y}\right)$ and wedge disclinations $\left(\Omega_{z}\right)$ for micropolar, nonlocal micropolar, and gradient micropolar elasticity. Once the internal length $\epsilon$ reduces, the densities of the nonlocal and gradient frameworks approach to the one in micropolar elasticity. Interestingly, different values of $\epsilon$ lead to qualitatively similar disclination densities in gradient version, while in the nonlocal version, higher $\epsilon$ creates a qualitatively different disclination distribution.

It is also observed that the dislocation and disclination densities of micropolar and gradient micropolar elasticity have qualitatively similar distribution, having opposite singularity at the crack tips compared to nonlocal micropolar elasticity. Similar trend was earlier reported once comparing classical and gradient elasticity theories with nonlocal elasticity [39,42-44].

The stress and couple stress fields can also be determined by convoluting the corresponding field of discrete line defect with its density. These fields are given in (62), (71), and (5.3) for micropolar, nonlocal micropolar, and gradient micropolar elasticity, respectively. Using these relations, the stress component $\sigma_{y y}$ and the couple

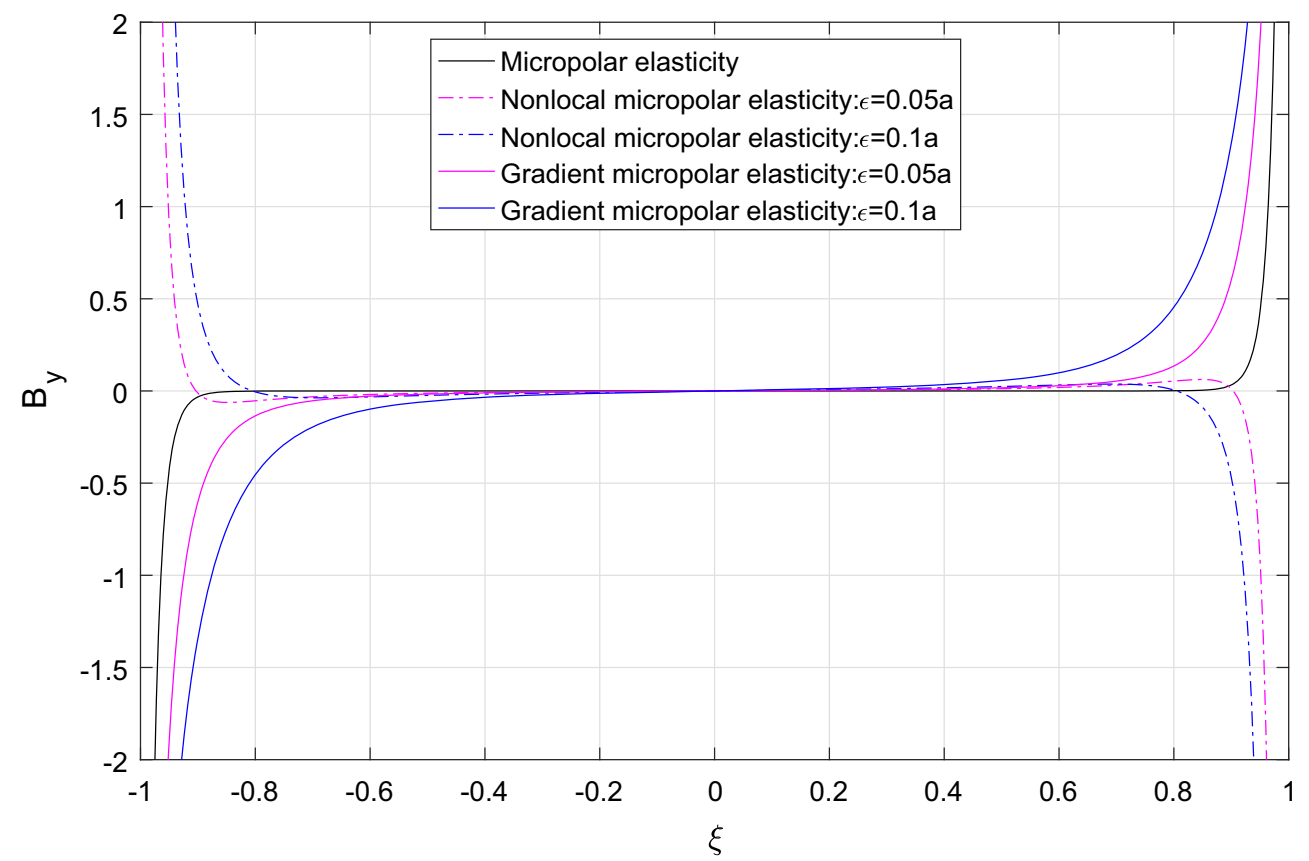

Fig. 3 Density of climb edge dislocation for loading case 1: $\sigma_{y y_{0}}$ (see online version for colors) 


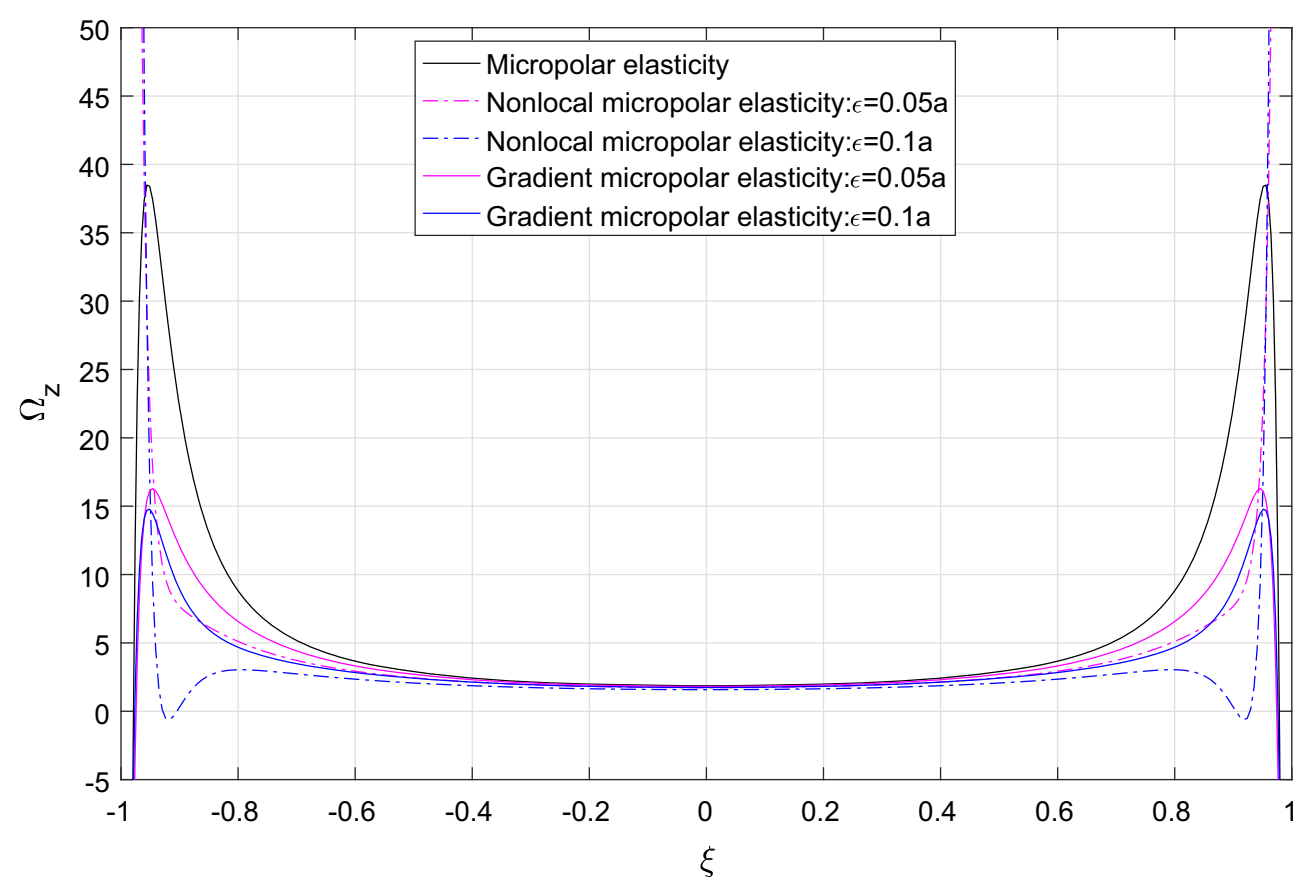

Fig. 4 Density of wedge disclination for loading case 1: $\sigma_{y y_{0}}$

stress component $\mu_{z y}$ are shown in Figs. 5 and 6, respectively. As expected, the singular fields of (classical) micropolar elasticity are regularized within nonlocal and gradient micropolar elasticity. Reducing the internal length $\epsilon$, the nonlocal and gradient fields approach to those of micropolar elasticity.

The distinction of the tractions (68) (in nonlocal micropolar elasticity) and (77) (in gradient micropolar elasticity) results in the difference in the field components of the nonlocal and gradient versions. In other words, we observe zero stress and couple stress fields along the crack only in the nonlocal micropolar elasticity.

To illustrate the regularization obtained within nonlocal micropolar elasticity, Fig. 7 depicts the stress and couple stress fields in the $x y$ plane at the crack tip. In this figure, the dimensionless field components are presented as the $z$-axis while colors have also been used to clarify the variations. It is noted that the crack is along $-a \leq x \leq a, y=0$ introduced earlier in (52). This figure depicts the contour plots of field components of nonlocal micropolar elasticity with $\epsilon=0.1 a$. Similar plots can be obtained for micropolar as well as gradient micropolar elasticity frameworks.

\subsection{Case 2}

The crack is now considered to be under remote loading $\sigma_{x y}^{\infty}=\sigma_{x y_{0}}$ while $\sigma_{y y}^{\infty}=0$ and $\mu_{z y}^{\infty}=0$. Considering the governing integral equations, only a distribution of glide edge dislocations can represent the crack. Similar to case 1 , the density of the glide edge dislocation is calculated for micropolar, nonlocal micropolar, and gradient micropolar elasticity using the corresponding governing equations. The result is depicted in Fig. 8. Similar to case 1, the distribution of glide edge dislocation within micropolar elasticity and gradient micropolar elasticity is qualitatively similar, while in nonlocal version the sign of the dislocation density at the crack tips is different.

The stress component $\sigma_{x y}$ is shown in Fig. 9, while no couple stress exists along the crack line in this case. Furthermore, Fig. 10 depicts the contour plots of the stress and couple stress fields in the $x y$ plane at the crack tip.

\subsection{Case 3}

As the last example, the crack is considered to be under remote loading $\mu_{z y}^{\infty}=\mu_{z y_{0}}$ while $\sigma_{x y}^{\infty}=0$ and $\sigma_{y y}^{\infty}=0$. In this case, a distribution of climb edge dislocation and wedge disclination can represent the crack. 


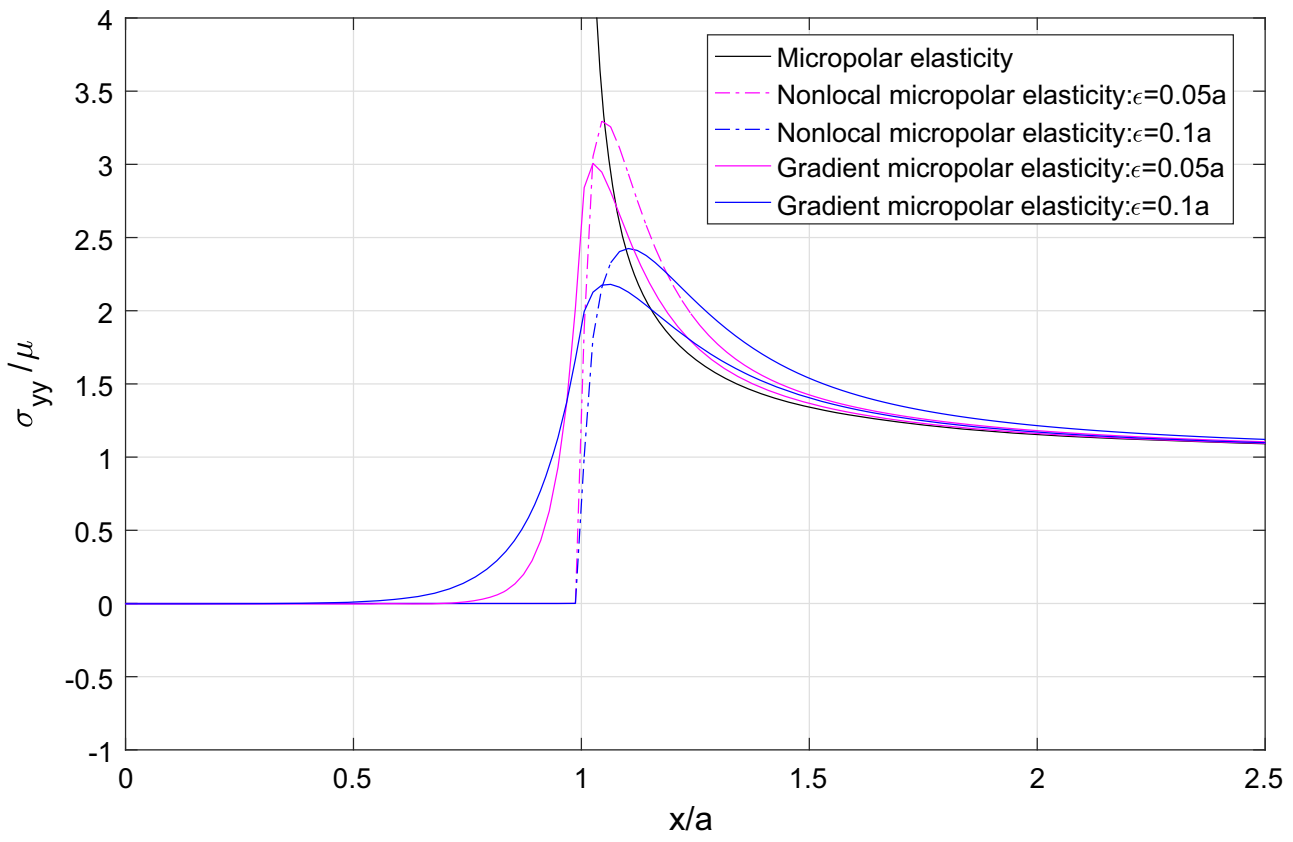

Fig. 5 Stress component $\sigma_{y y}$ along the crack line $y=0$ for loading case 1: $\sigma_{y y_{0}}$

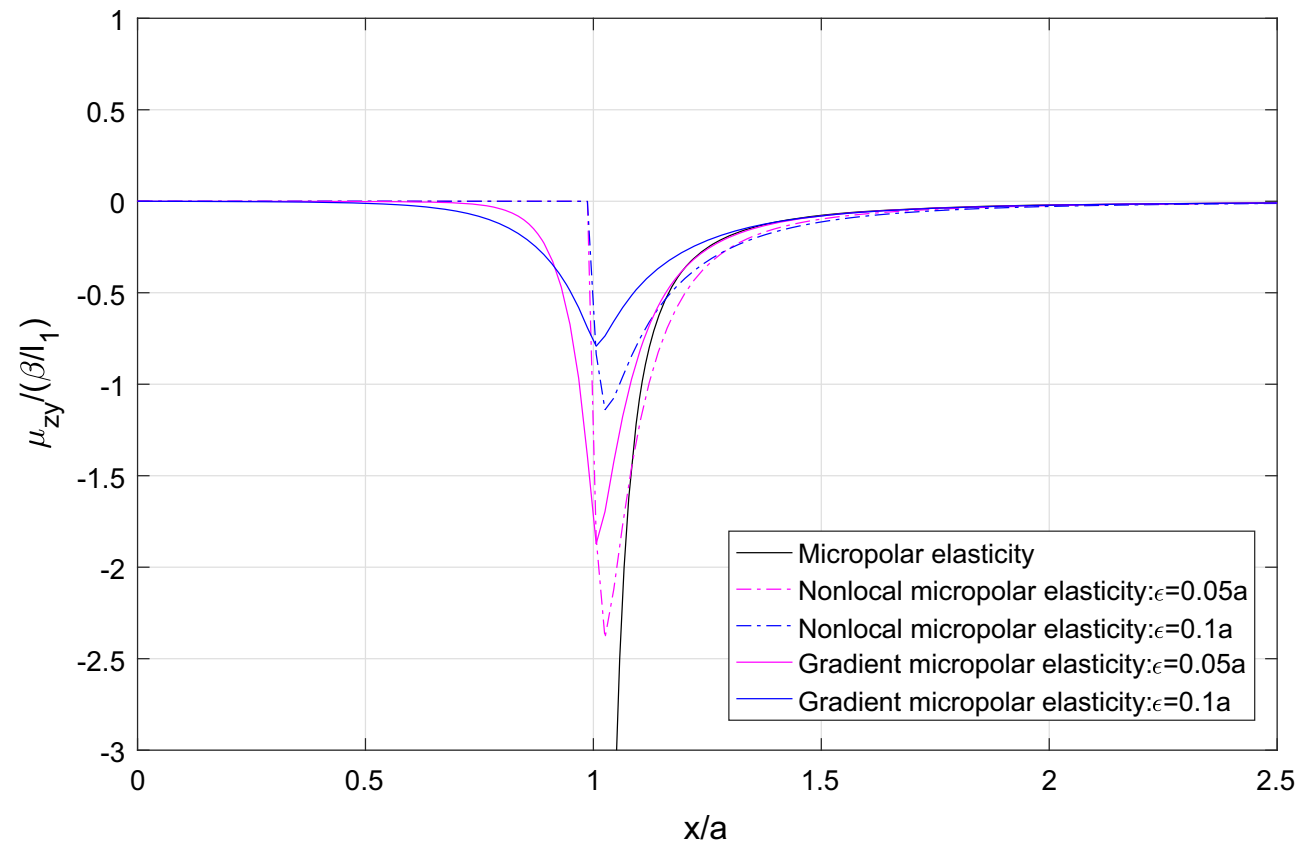

Fig. 6 Couple stress component $\mu_{z y}$ along the crack line $y=0$ for loading case 1: $\sigma_{y y_{0}}$

Using the formulation developed in the previous sections, the densities of the climb edge dislocations and wedge disclinations for micropolar, nonlocal micropolar and gradient micropolar elasticity are determined and depicted in Figs. 11 and 12, respectively.

Furthermore, the stress component $\sigma_{y y}$ and the couple stress component $\mu_{z y}$ are shown in Figs. 13 and 14, respectively. Finally, Fig. 15 depicts the stress and couple stress fields in the $x y$ plane at the crack tip for loading case 3. 

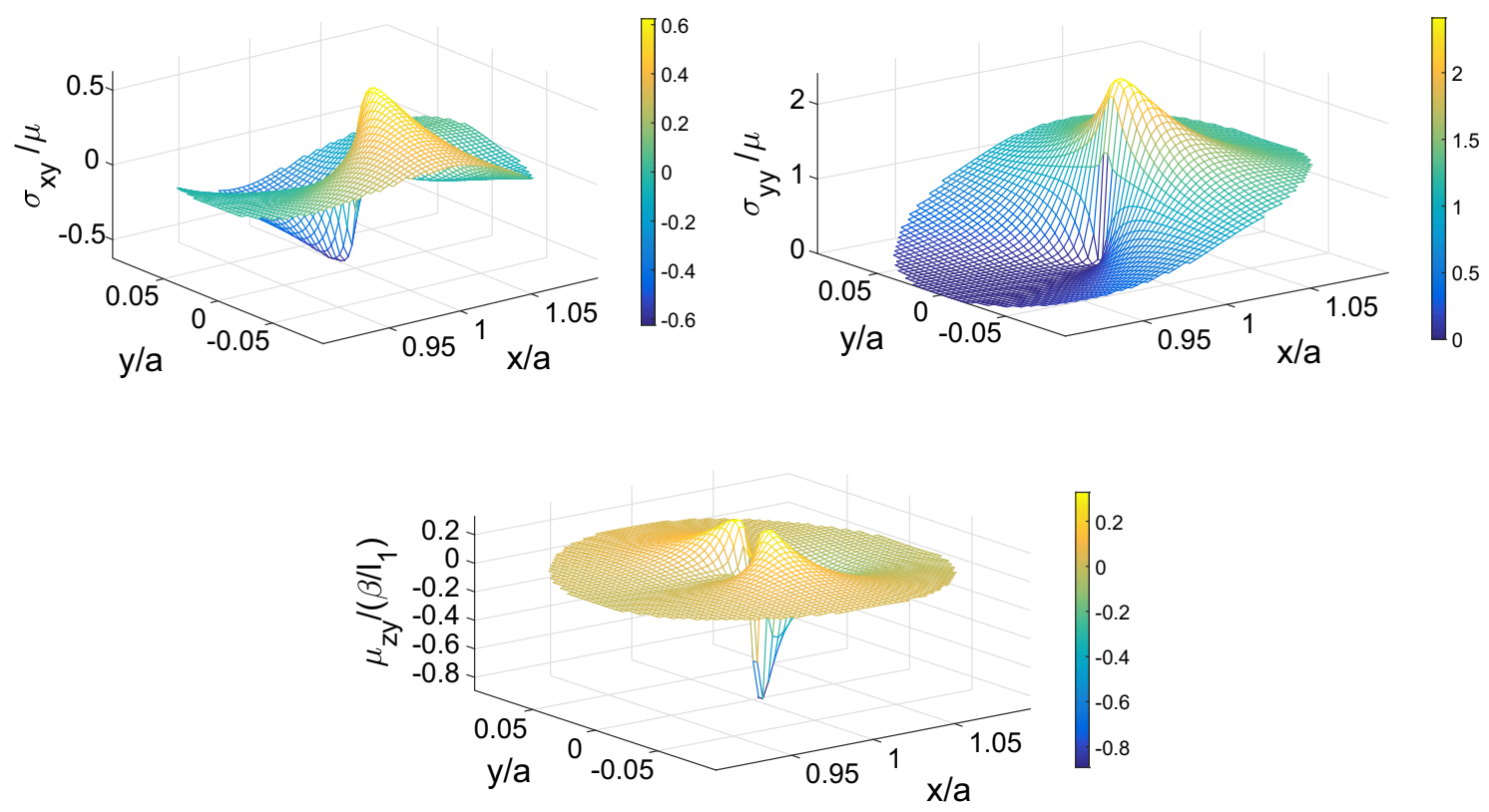

Fig. 7 Stress and couple stress fields within nonlocal micropolar elasticity $(\epsilon=0.1 a)$ around the crack tip for loading case 1

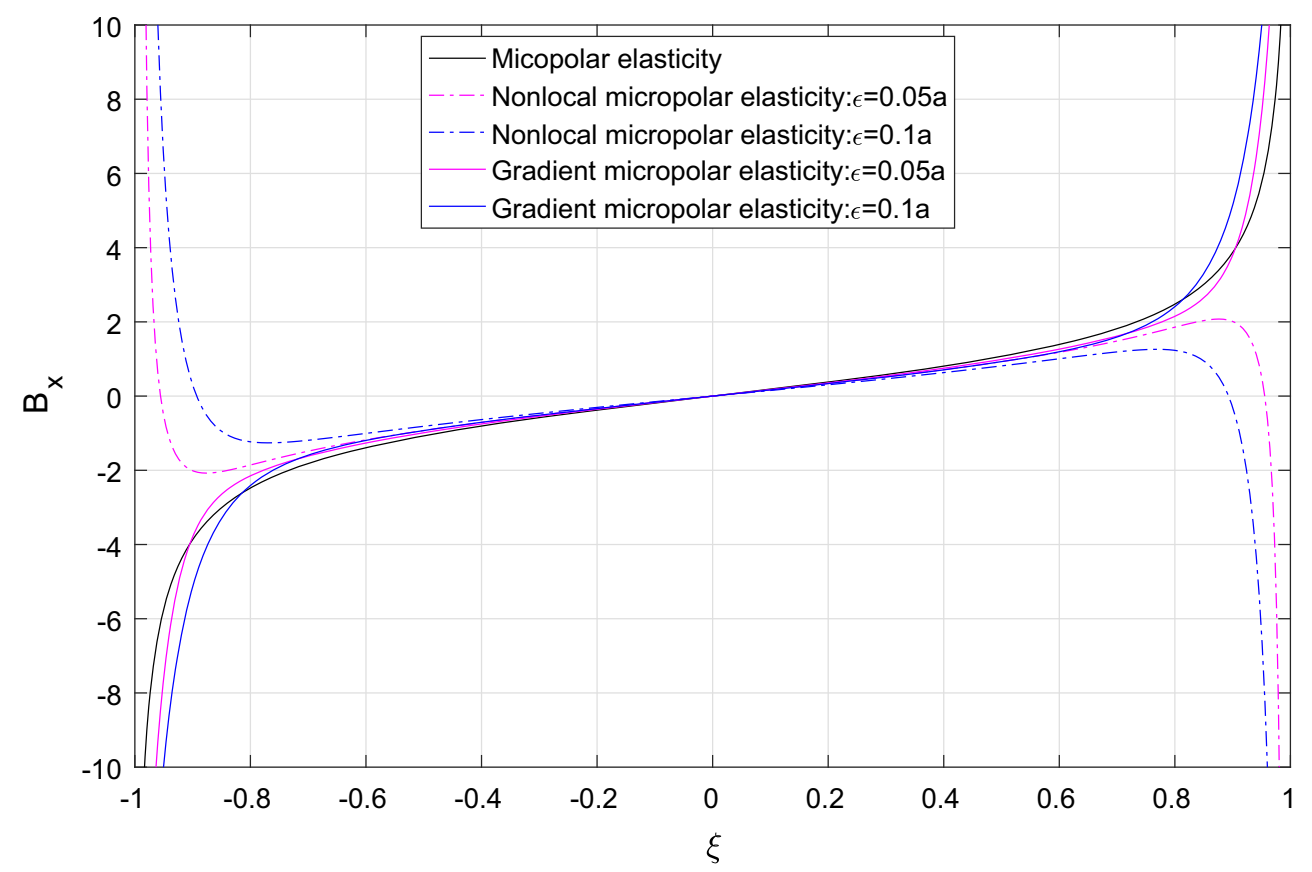

Fig. 8 Density of glide edge dislocation for loading case 2: $\sigma_{x y_{0}}$

\section{Discussions}

Generalized continua address materials with internal length scales. In the case of higher-order continua, once studying granular materials [37], the internal length scales can be in the order of the microstructural scale. In this case, the predictions of generalized continua can be compared with results from atomistic simulation. For such comparison, the classical stress definitions in atomistic simulation (such as Hardy stress) should be extended to continuum definitions for stress and couple stress in atomistic simulation [62]. The atomic model is shown to be consistent with the higher-order continuum model [7] while the implementation of the atomistic simulation to 


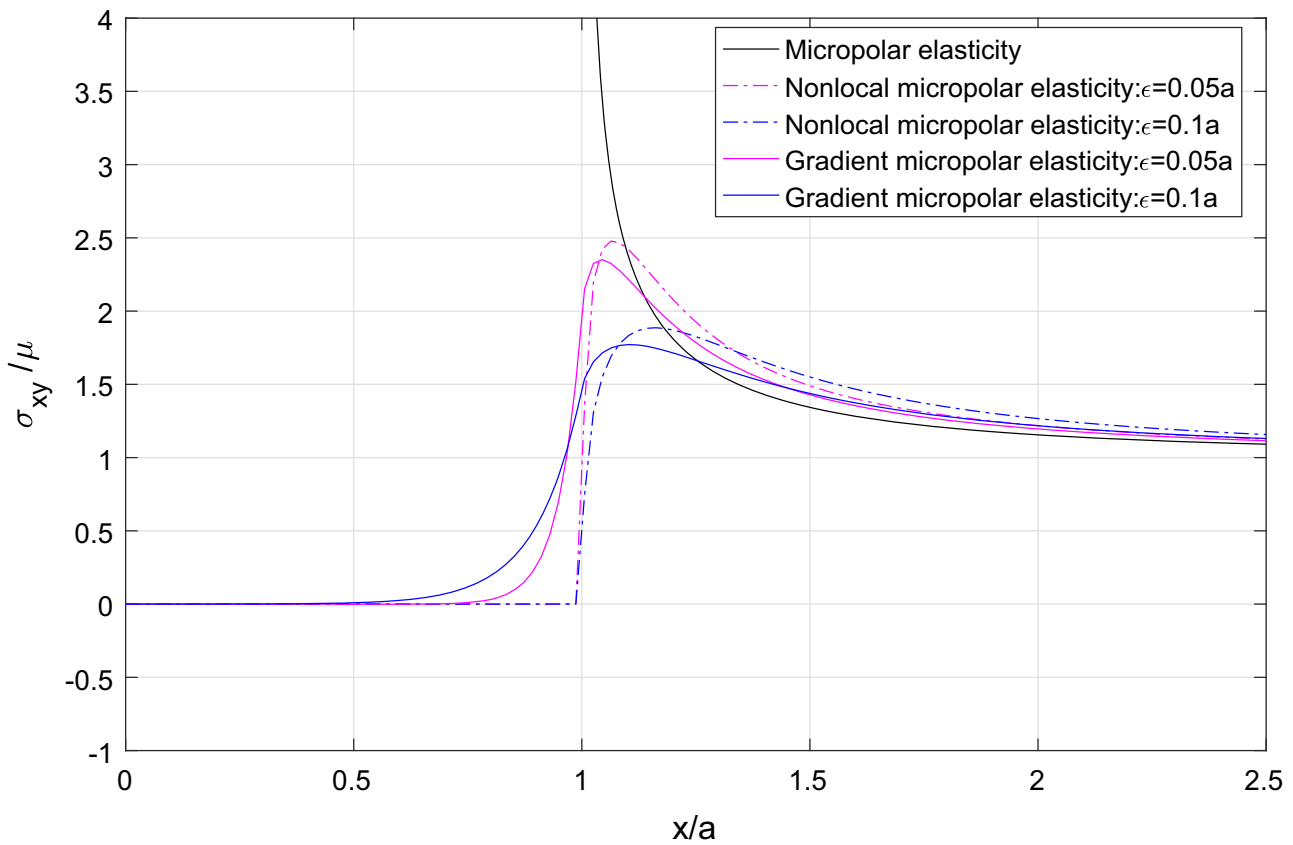

Fig. 9 Stress component $\sigma_{x y}$ along the crack line $y=0$ for loading case 2: $\sigma_{x y_{0}}$
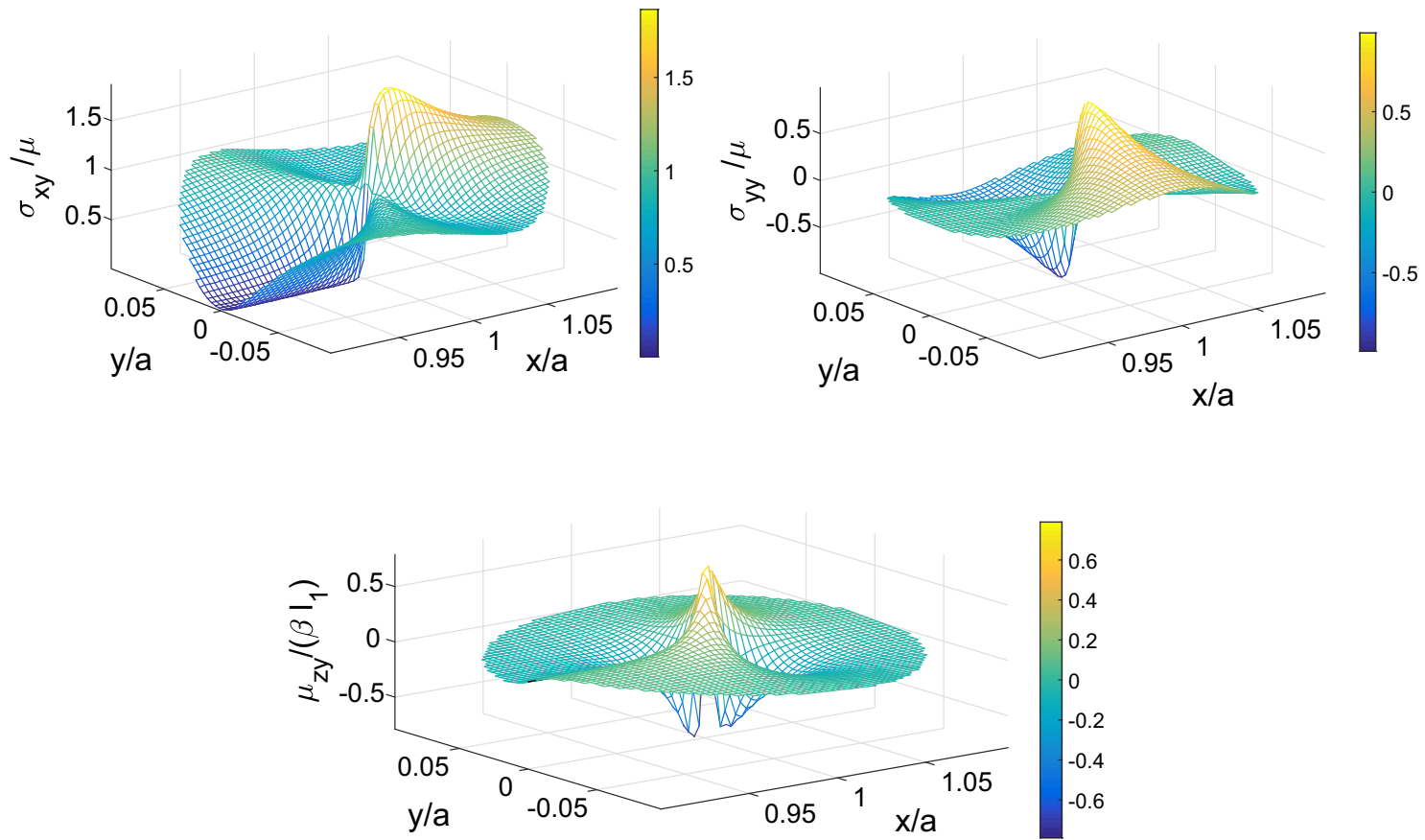

Fig. 10 Stress and couple stress fields within nonlocal micropolar elasticity $(\epsilon=0.1 a)$ around the crack tip for loading case 2

defective polar materials is yet untouched. However, it is well shown that the classical stress intensity factor fails to describe fracture below a critical singular field of 2-3 nm for materials such as silicon, i.e., the breakdown of continuum fracture mechanics at small scales [50]. Furthermore, the classical finite element informed atomistic simulation [45] may be extended to connect corresponding higher-order continua and atomistic simulation.

Interestingly, these length scales may be of much higher order once looking into cellular materials. Cancellous bone is one of the bone tissues which can be considered as a cellular solid. Goda et al. [17] studied cracked 


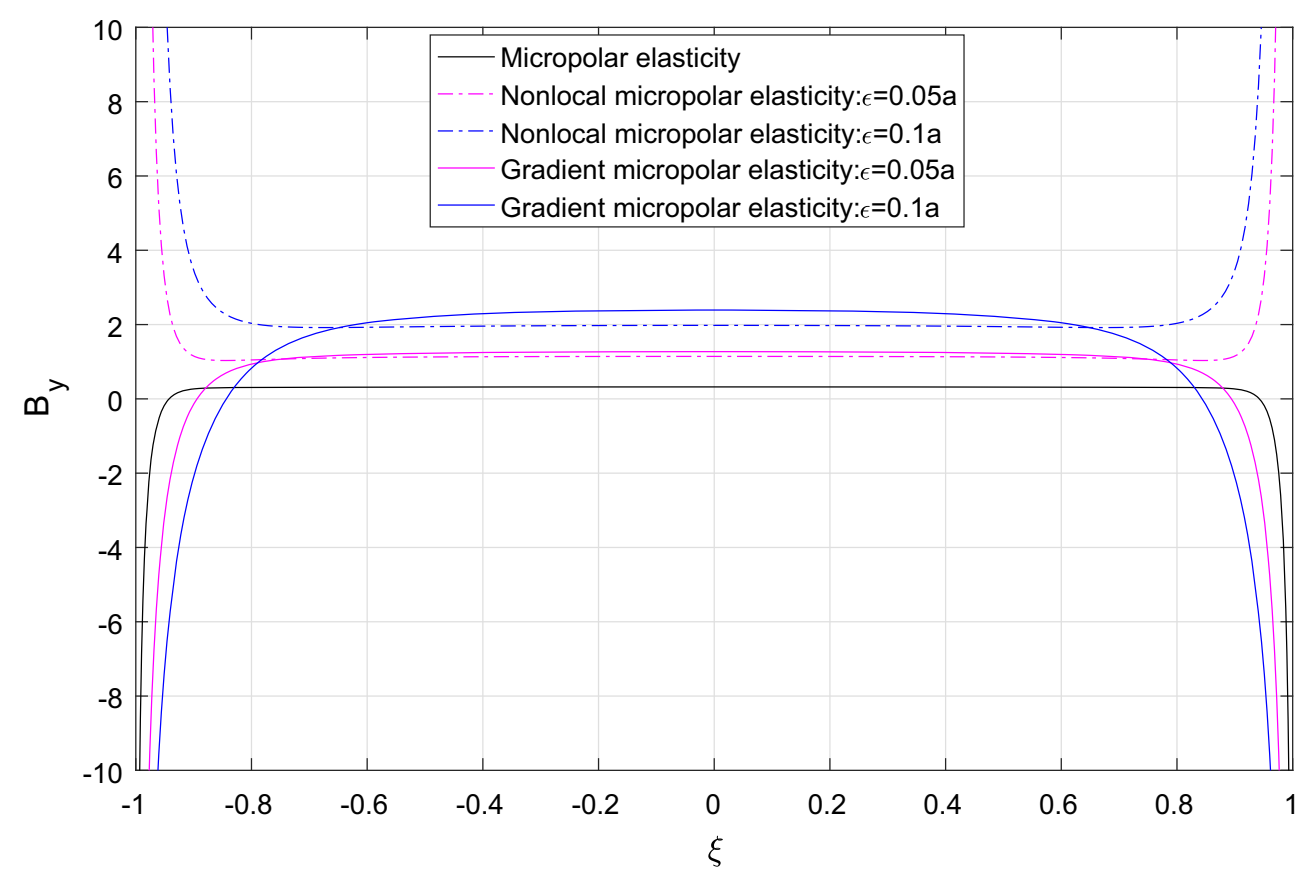

Fig. 11 Density of climb edge dislocation for loading case 3: $\mu_{z y_{0}}$

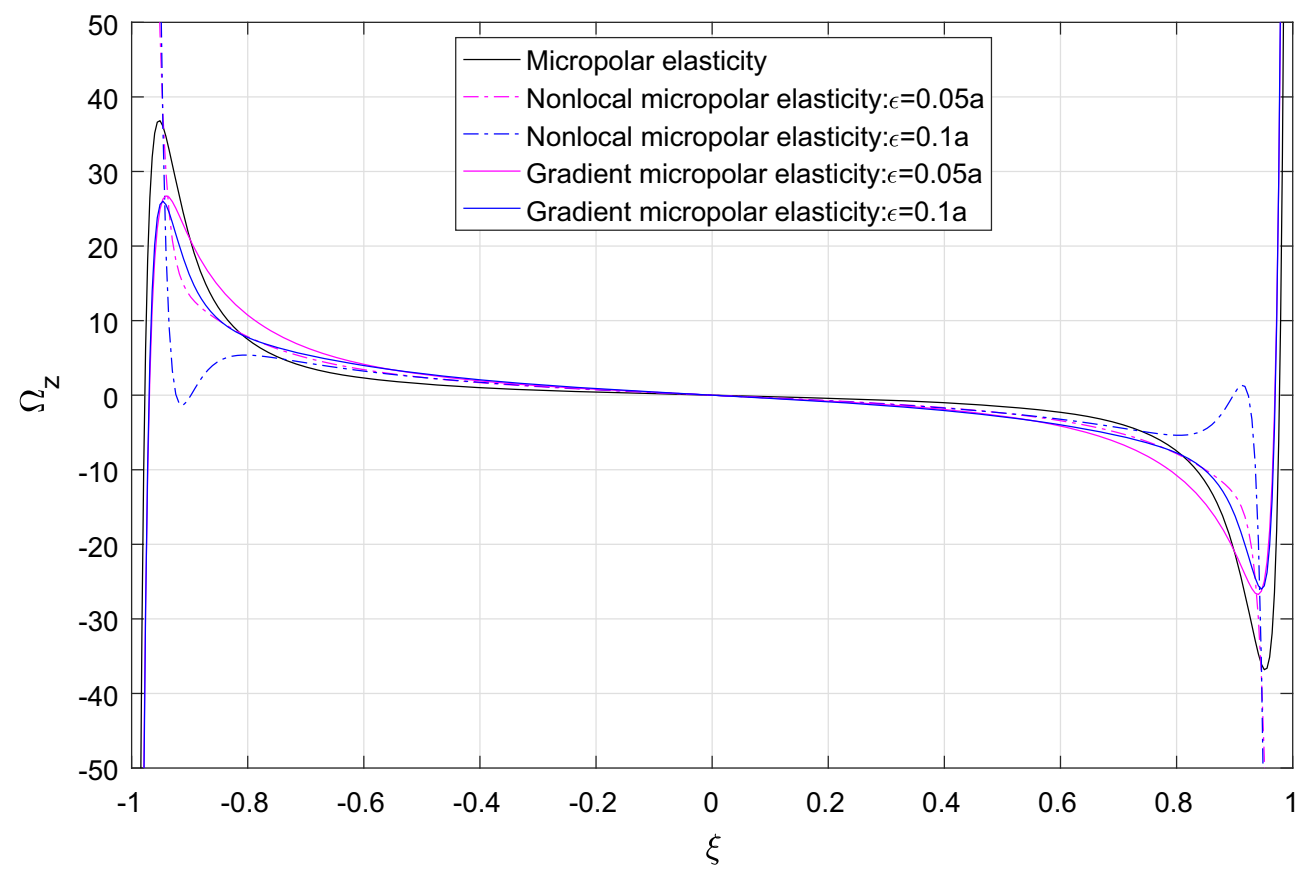

Fig. 12 Density of wedge disclination for loading case 3: $\mu_{z y_{0}}$

cancellous bone based on an effective micropolar model reporting its regularizing effect in comparison with classical elasticity (Cauchy continuum).

The formulation and the numerical results presented in the previous sections report the near-core behavior of line defects and near-tip behavior of complex defects in the solids for which material particles can displace as well as rotate. Having fully identified the defects fields, one can consider the simple and complex defects as design parameters. In other words, instead of simply avoiding the defects, a realistic prediction of the defective material behavior can lead to advanced tools for engineering the defects. 


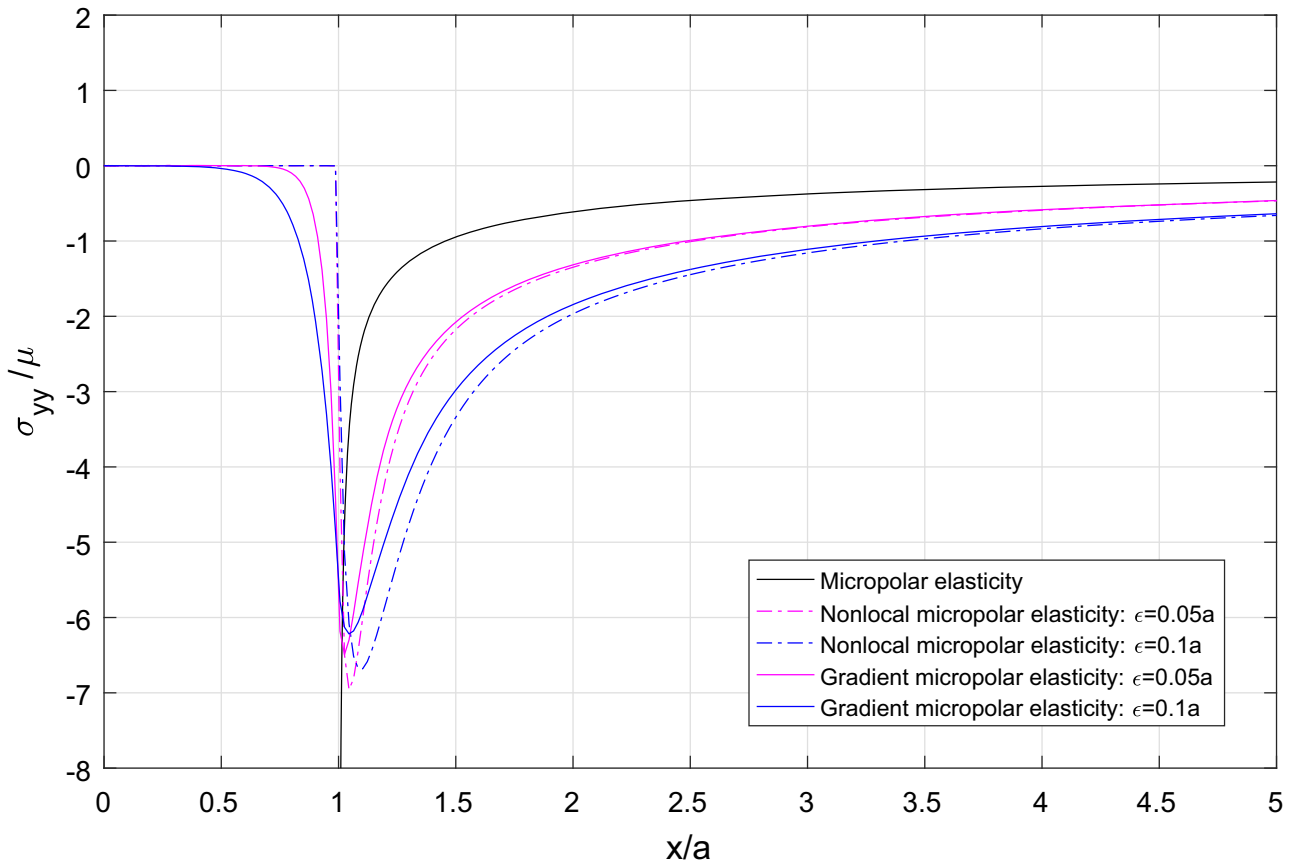

Fig. 13 Stress component $\sigma_{y y}$ along the crack line $y=0$ for loading case 3: $\mu_{z y_{0}}$

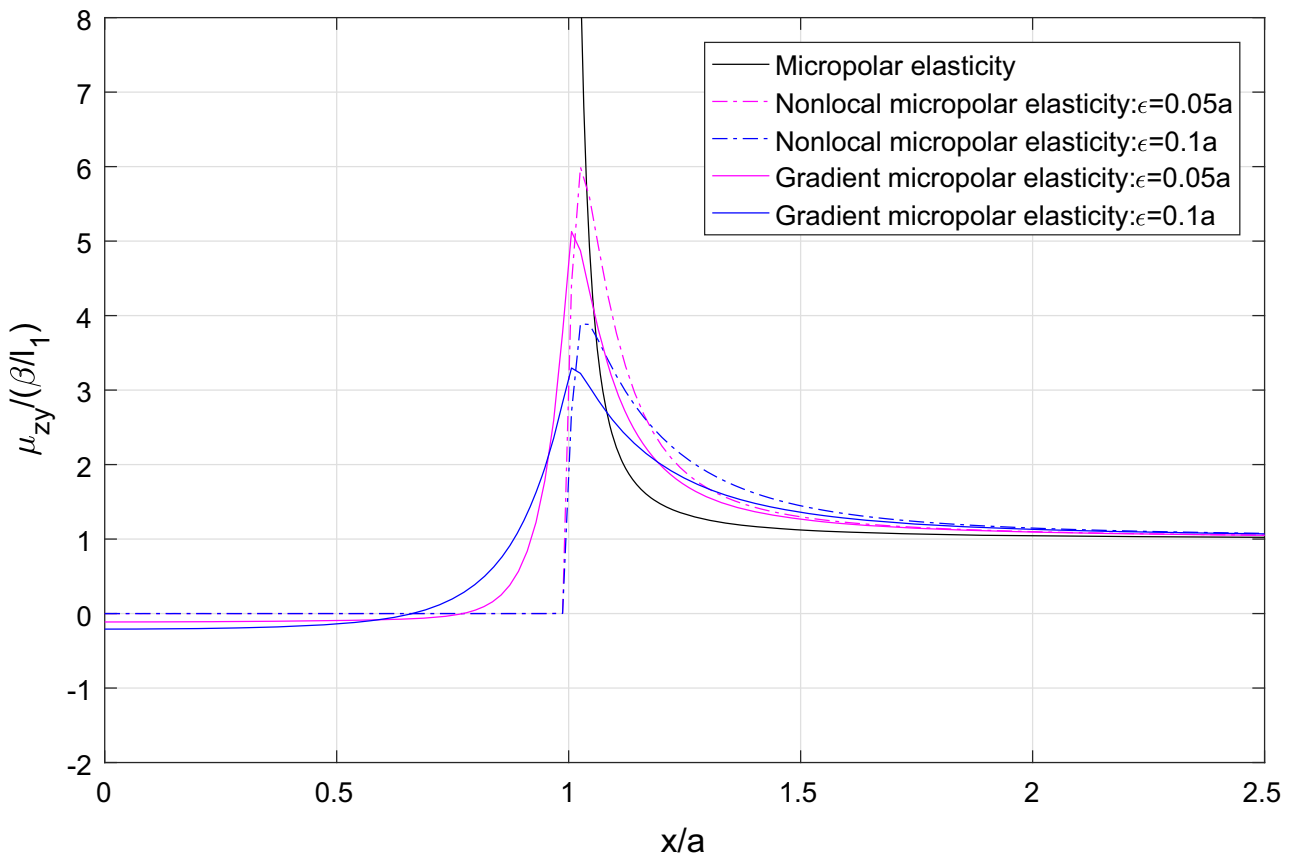

Fig. 14 Couple stress component $\mu_{z y}$ along the crack line $y=0$ for loading case $3: \mu_{z y_{0}}$

\section{Conclusion}

Micropolar continuum theory has proved its potential for the analysis of materials, such as granular materials, where material points possess both position and orientation. To fully implement this continuum theory in the design and analysis, we looked into the presence of simple and complex defects in such media. We employed a suitable collection of line defects to study complex defects within extended continua. Higher-order frameworks (i.e., micropolar elasticity) and simultaneously higher-order and higher-grade theories (i.e., nonlocal microp- 

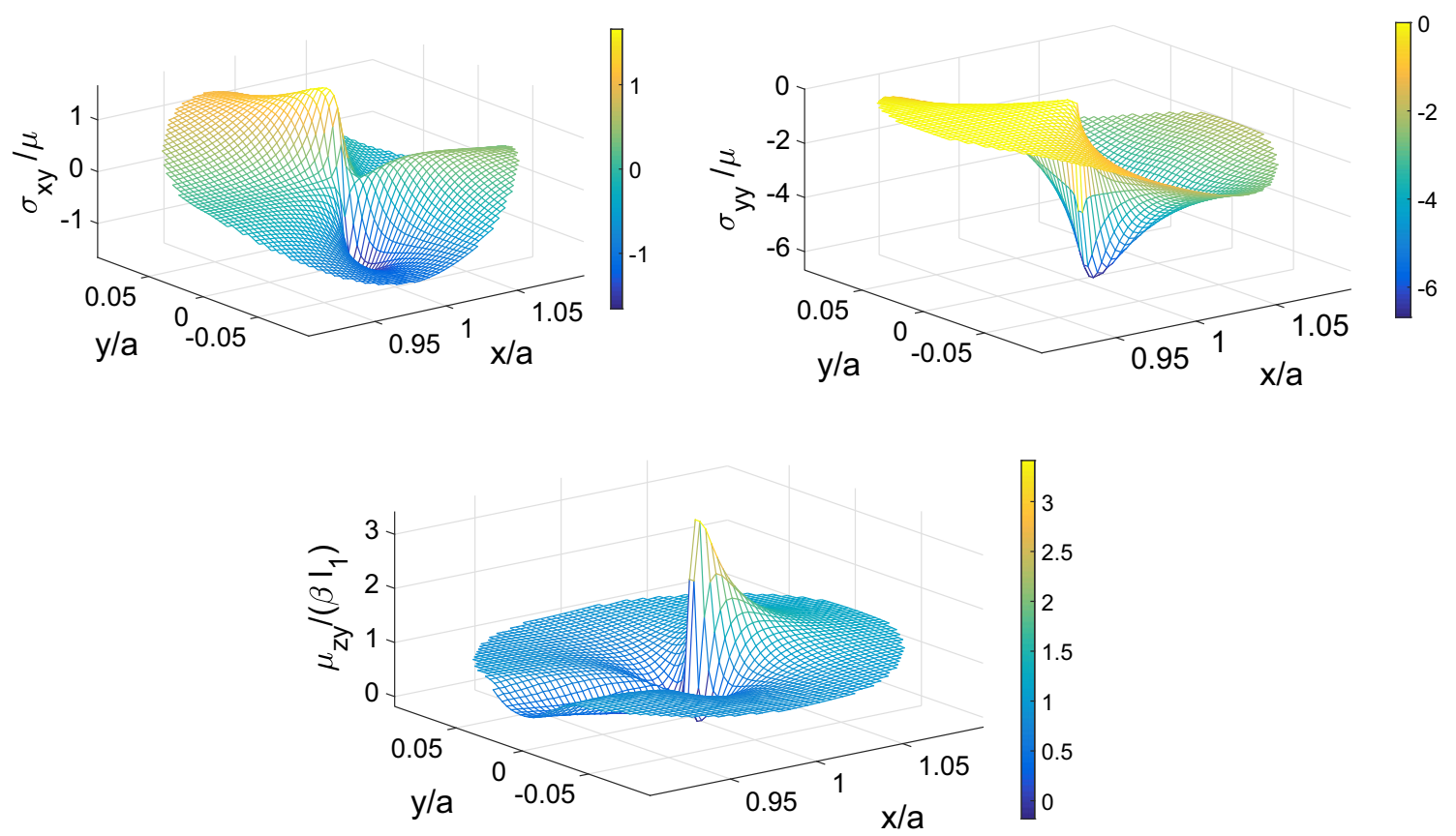

Fig. 15 Stress and couple stress fields within nonlocal micropolar elasticity $(\epsilon=0.1 a)$ around the crack tip for loading case 3

olar and gradient micropolar elasticity) were addressed. Starting from a collection of line defects including dislocations and disclinations, a systematic approach was developed to identify consistent line defects to represent the complex defect. These line defects were then employed to construct the cracks undergoing plane strain. The analysis of cracks with antiplane strain is left to another study.

Line defects within micropolar elasticity contain singularity at the core. This singularity was regularized within nonlocal and gradient version of micropolar elasticity. The crack was represented as distributions of line defects. Consequently, the fields at the crack tip are singular within micropolar elasticity and nonsingular within nonlocal and gradient micropolar elasticity theories.

Planes with three different load cases, i.e., planes under $\sigma_{x y 0}, \sigma_{y y 0}$, and $\mu_{z y 0}$, were studied separately, and the defect densities as well as stress and couple stress fields were determined. Any combination of loading can be analyzed by superposing these load cases. It is noted that the types of the discrete line defects which could represent the crack in load cases 1 and 2 are consistent with those reported by Dyskin and Pasternak [11]. In that paper, symmetry conditions were used to identify the suitable line defects, while here the types of the defects were derived automatically simply by considering the consistent deformation and rotation discontinuities and the corresponding traction conditions.

The success of this approach promises a comprehensive method for the analysis of complex defects based on simple defects within generalized continua. This method benefits from the extensive literature on the analysis of simple defects. This complements the ongoing investigations on the dislocation-based plasticity theories to aim for a unified defect-based plasticity and fracture mechanics.

Acknowledgements S. M. M. acknowledges financial support from the Starting Grant of the Swedish Research Council (201803636).

Open Access This article is distributed under the terms of the Creative Commons Attribution 4.0 International License (http:// creativecommons.org/licenses/by/4.0/), which permits unrestricted use, distribution, and reproduction in any medium, provided you give appropriate credit to the original author(s) and the source, provide a link to the Creative Commons license, and indicate if changes were made.

\section{References}

1. Acharya, A.: New inroads in an old subject: plasticity, from around the atomic to the macroscopic scale. J. Mech. Phys. Solids 58, 766-778 (2010) 
2. Atkinson, E., Leppington, R.: The effect of couple stresses on the tip of a crack. Int. J. Solids Struct. 13, 1103-1122 (1967)

3. Atroshchenko, E., Bordas, P.A.: Fundamental solutions and dual boundary element methods for fracture in plane Cosserat elasticity. Proc. R. Soc. A Math. Phys. Eng. Sci. 471, 20150216 (2015)

4. Bilby, B.A., Eshelby, J.D.: Dislocations and the theory of fracture. In: Liebowitz, H. (ed.) Fracture, An Advanced Treatise, pp. 100-182. Academic Press, New York (1968); reprinted in Collected Works of Eshelby, J.D., Markenscoff, X., Gupta, A., (Eds.), pp. 861-902. Springer, Dordrecht (2006)

5. Bueckner, H.F.: Mechanics of Fracture I, pp. 239-314. Noordhoff, Leyden (1973)

6. Chen, J.Y., Huang, Y., Ortiz, M.: Fracture analysis of cellular materials: a strain gradient model. J. Mech. Phys. Solids 46, 789-828 (1998)

7. Chen, Y., Lee, J.D.: Connecting molecular dynamics to micromorphic theory. (I). Instantaneous and averaged mechanical variables. Physica A 322, 359-376 (2003)

8. Cosserat, E., Cosserat, F.: Théorie des corps déformables. Herman et Fils, Paris (1909)

9. deWit, R.: Theory of disclinations IV. Straight disclinations. J. Res. Natl. Inst. Stand. 77A, 607-658 (1973)

10. Diegele, E., Elsaber, R., Tsakmakis, C.: Linear micropolar elastic crack-tip fields under mixed mode loading conditions. Int. J. Fract. 129, 309-339 (2004)

11. Dyskin, A.V., Pasternak, E.: Asymptotic analysis of fracture propagation in materials with rotating particles. Eng. Fract. Mech. 150, 1-18 (2015)

12. Eremeyev, V.A., Lebedev, L.P., Altenbach, H.: Foundations of Micropolar Mechanics. Springer, New York (2013)

13. Ericksen, J.L., Truesdell, C.: Exact theory of stress and strain in rods and shells. Arch. Ration. Mech. Anal. 1, 295-323 (1957)

14. Eringen, A.C.: Microcontinuum Field Theories I: Foundations and Solids. Springer, New York (1999)

15. Eringen, A.C.: Nonlocal Continuum Field Theories. Springer, New York (2002)

16. Neff, P., Ghiba, I.D., Madeo, A., Placidi, L., Rosi, G.: A unifying perspective: the relaxed linear micromorphic continuum. Contin. Mech. Thermodyn. 26, 639-681 (2014)

17. Goda, I., Assidi, M., Belouettar, S., Ganghoffer, J.F.: A micropolar anisotropic constitutive model of cancellous bone from discrete homogenization. J. Mech. Behav. Biomed. Mater. 16, 87-108 (2012)

18. Gourgiotis, P.A., Georgiadis, H.G.: Distributed dislocation approach for cracks in couple-stress elasticity: shear modes. Int. J. Fract. 147, 83-102 (2007)

19. Gourgiotis, P.A., Georgiadis, H.G.: An approach based on distributed dislocations and disclinations for crack problems in couple-stress elasticity. Int. J. Solids Struct. 45, 5521-5539 (2007)

20. Hassanpour, S., Heppler, G.R.: Micropolar elasticity theory: a survey of linear isotropic equations, representative notations, and experimental investigations. Math. Mech. Solids 22, 224-242 (2017)

21. Hills, D., Kelly, P., Dai, D., Korsunsky, A.: Solution of Crack Problems: The Distributed Dislocation Technique. Springer, New York (1996)

22. Hirth, J.P., Lothe, J.: Theory of Dislocations, 2nd edn. Wiley, New York (1982)

23. Hochrainer, T., Sandfeld, S., Zaiser, M., Gumbsch, P.: Continuum dislocation dynamics: towards a physical theory of crystal plasticity. J. Mech. Phys. Solids 63, 167-178 (2014)

24. Kapiturova, M., Gracie, R., Potapenko, S.: Simulation of cracks in a Cosserat medium using the extended finite element method. Math. Mech. Solids 21, 621-635 (2016)

25. Kleman, M., Friedel, J.: Disclinations, dislocations, and continuous defects: a reappraisal. Rev. Mod. Phys. 80, 61-115 (2008)

26. Lakes, R.: Size effects and micromechanics of a porous solid. J. Mater. Sci. 18, 2572-2580 (1983)

27. Lazar, M., Kirchner, H.O.K.: Cosserat (micropolar) elasticity in Stroh form. Int. J. Solids Struct. 42, 5377-5398 (2005)

28. Lazar, M., Kirchner, H.O.K.: The Eshelby tensor in nonlocal elasticity and in nonlocal micropolar elasticity. J. Mech. Mater. Struct. 1, 325-337 (2006)

29. Lazar, M., Maugin, G.A.: Defects in gradient micropolar elasticity-I: screw dislocation. J. Mech. Phys. Solids 52, 2263-2284 (2004)

30. Lazar, M., Maugin, G.A.: Defects in gradient micropolar elasticity-II: edge dislocation and wedge disclination. J. Mech. Phys. Solids 52, 2285-2307 (2004)

31. Lazar, M., Maugin, G.A., Aifantis, E.C.: On dislocations in a special class of generalized elasticity. Phys. Status Solidi B 242, 2365-2390 (2005)

32. Lazar, M., Maugin, G.A.: On microcontinuum field theories: the Eshelby stress tensor and incompatibility conditions. Philos. Mag. 87, 3853-3870 (2007)

33. Li, Y.D., Lee, K.Y.: Fracture analysis in micropolar elasticity: anti-plane crack. Int. J. Fract. 152, 163-168 (2008)

34. Li, Y.D., Lee, K.Y.: Fracture analysis in micropolar elasticity: mode-I crack. Int. J. Fract. 156, 179-184 (2008)

35. Lubarda, V.A.: The effects of couple stresses on dislocation strain energy. Int. J. Solids Struct. 40, 3807-3826 (2003)

36. Maugin, G.A.: Continuum Mechanics Through the Twentieth Century: A Concise Historical Perspective. Springer, New York (2013)

37. Misra, M., Poorsolhjouy, P.: Grain- and macro-scale kinematics for granular micromechanics based small deformation micromorphic continuum model. Mech. Res. Commun. 81, 1-6 (2017)

38. Mousavi, S.M., Paavola, J., Baroudi, J.: Distributed nonsingular dislocation technique for cracks in strain gradient elasticity. J. Mech. Behav. Mater. 23, 47-48 (2014)

39. Mousavi, S.M., Lazar, M.: Distributed dislocation technique for cracks based on non-singular dislocations in nonlocal elasticity of Helmholtz type. Eng. Fract. Mech. 136, 79-95 (2015)

40. Mousavi, S.M., Korsunsky, A.M.: Non-singular fracture theory within nonlocal anisotropic elasticity. Mater. Des. 88, 854-861 (2015)

41. Mousavi, S.M., Aifantis, E.C.: A note on dislocation-based mode III gradient elastic fracture mechanics. J. Mech. Behav. Mater. 24, 115-119 (2015)

42. Mousavi, S.M., Aifantis, E.C.: Dislocation-based gradient elastic fracture mechanics for in-plane analysis of cracks. Int. J. Fract. 202, 93-110 (2016) 
43. Mousavi, S.M.: Dislocation-based fracture mechanics within nonlocal and gradient elasticity of bi-Helmholtz type-Part I: antiplane analysis. Int. J. Solids Struct. 87, 222-235 (2016)

44. Mousavi, S.M.: Dislocation-based fracture mechanics within nonlocal and gradient elasticity of bi-Helmholtz type-Part II: inplane analysis. Int. J. Solids Struct. 92, 105-120 (2016)

45. Möller, J., Prakash, A., Bitzek, E.: FE2AT-finite element informed atomistic simulations. Model. Simul. Mater. Sci. Eng. 21, 055011 (2013)

46. Nowacki, W.: On discrete dislocations in micropolar elasticity. Arch. Mech. 26, 3-11 (1974)

47. Nowacki, W.: Theory of Asymmetric Elasticity. Pergamon Press, Oxford (1986)

48. Po, G., Lazar, M., Seif, D., Ghoniem, N.: Singularity-free dislocation dynamics with strain gradient elasticity. J. Mech. Phys. Solids 68, 161-178 (2014)

49. Povstenko, Y.Z.: Stress functions for continua with couple stresses. J. Elast. 36, 99-116 (1994)

50. Shimada, T., Ouchi, K., Chihara, Y., Kitamura, T.: Validity of linear elasticity in the crack-tip region of ideal brittle solids. Sci. Rep. 5, 8596 (2015)

51. Shmoylova, E., Potapenko, S., Rothenburg, L.: Boundary element analysis of stress distribution around a crack in plane micropolar elasticity. Int. J. Eng. Sci. 45, 199-209 (2007)

52. Sternberg, E., Muki, R.: The effect of couple-stresses on the stress concentration around a crack. Int. J. Solids Struct. 3 , 69-95 (1967)

53. Tornabene, F., Fantuzzi, N., Bacciocchi, M.: Mechanical behaviour of composite Cosserat solids in elastic problems with holes and discontinuities. Compos. Struct. 179, 468-481 (2017)

54. Toupin, R.A.: Elastic materials with couple-stresses. Arch. Ration. Mech. Anal. 11, 385-414 (1962)

55. Toupin, R.A.: Theories of elasticity with couple-stress. Arch. Ration. Mech. Anal. 17, 85-112 (1964)

56. Warren, W.E., Byskov, E.: A general solution to some plane problems of micropolar elasticity. Eur. J. Mech. A Solids 27, $18-27(2008)$

57. Weertman, J.: Dislocation Based Fracture Mechanics. World Scientific, Singapore (1996)

58. Yavari, A., Sarkani, S., Moyer, E.T.: On fractal cracks in micropolar elastic solids. J. Appl. Mech. 69, 45-54 (2002)

59. Yu, H., Sumigawa, T., Kitamura, T.: A domain-independent interaction integral for linear elastic fracture analysis of micropolar materials. Mech. Mater. 74, 1-13 (2014)

60. Yu, H., Sumigawa, T., Kitamura, T., Kuna, M.: Domain-independent I-integrals for force and couple stress intensity factor evaluations of a crack in micropolar thermoelastic. Int. J. Solids Struct. 100-101, 470-484 (2016)

61. Zhang, C., Acharya, A.: On the relevance of generalized disclinations in defect mechanics. J. Mech. Phys. Solids 119, $188-223(2018)$

62. Zimmerman, J.A., Jones, R.E., Klein, P.A., Bammann, D.J., Webb, E.B., Hoyt, J.J.: Continuum definitions for stress in atomistic simulation. SAND Report, Sandia National Laboratory, SAND2002-8608 (2002)

63. Zubov, L.M.: Continuously distributed dislocations and disclinations in nonlinearly elastic micropolar media. Dokl. Phys. 49, 308-310 (2004)

Publisher's Note Springer Nature remains neutral with regard to jurisdictional claims in published maps and institutional affiliations. 